\title{
Generalized Walén tests through Alfvén waves and rotational discontinuities using electron flow velocities
}

\author{
J. D. Scudder, ${ }^{1}$ P. A. Puhl-Quinn, ${ }^{1}$ F. S. Mozer, ${ }^{2}$ K. W. Ogilvie, ${ }^{3}$ and \\ C. T. Russell ${ }^{4}$
}

\begin{abstract}
A new formulation of the Walén test [Walén, 1944] to locate rotational discontinuities (RD) or Alfvén wave trains is constructed that is more appropriate for use within and across the current-carrying layers of these structures. This generalized test is to be distinguished from the traditional rigorous jump condition that is only appropriate for connecting asymptotic, current free states on either side of the discontinuity or wave train. The generalized test is most simply formulated as a vector difference equation relating changes in the electron flow velocity vector and changes of the magnetic field vector, $\Delta \mathbf{U}_{e}(x)=\alpha_{e} \Delta \mathbf{B}(x)$ with a prescribed scalar constant of proportionality. For an electron proton plasma this paper shows that $\alpha_{e}$ is precisely the same constant of the jump condition that has long been in use. Small corrections to $\alpha_{e}$ are computed for minor helium populations. This new formulation is used with Polar plasma, E, and $\mathbf{B}$ measurements to search for rotational discontinuities at the magnetopause. Of the 44 cases where electrons gave acceptable theoretical correlations and coefficients $\alpha_{e}$ predicted by the theory, nearly $95 \%$ of the simultaneous ion fits gave corresponding values of $\alpha_{i}$ that were smaller in magnitude than implied by simple ideal MHD jump conditions. Possible explanations for this discrepancy are discussed. In addition, these data have been used to provide the first empirical evidence of the importance of the electron pressure gradient in these RD current layers and represent the first direct determination of the sizes $E_{n}^{\mathrm{HT}} \simeq 0.3-5 \mathrm{mV} / \mathrm{m}$ of the deHoffmann-Teller electric field in RD current layers at the magnetopause.
\end{abstract}

\section{Introduction}

Searches for rotational discontinuities have either used magnetometer data and the minimum variance approach to certify a normal component or tests that involve both particle and field measurements. One of the plasma and field tests used to certify that fluctuations are Alfvénic is based on the finite amplitude relation [Walén, 1944] of ideal MHD between the magnetic field $\mathbf{B}$ and the center of mass fluid velocity $\mathbf{U}_{c m}$ and total mass density $\rho_{\Sigma}$ of the form

$$
\left[\mathbf{U}_{c m}\right]= \pm \alpha\left[\frac{\mathbf{B}}{\rho_{\Sigma}}\right],
$$

where $[z] \equiv z^{+\infty}-z^{-\infty}$ stands for the asymptotic jumps in the quantities well removed from the shear in the magnetic field and the flows. Many searches for rotational discontinuities in space plasmas attempt to test magnetometer and ion plasma flows as a proxy for $\mathbf{U}_{c m}$ for the required vectorial parallelism of (1) and the scaling of the proportionality constant itself in the Walén relation summarized in (1). The size of $\alpha$ is prescribed by the theory and is given by the two relation-

\footnotetext{
${ }^{1}$ Department of Physics and Astronomy, University of Iowa, Iowa City.

${ }^{2}$ Space Sciences Laboratory, University of California, Berkeley.

${ }^{3}$ Laboratory for Extraterrestrial Physics, NASA Goddard Space Flight Center, Greenbelt, Maryland.

${ }^{4}$ Institute of Geophysics and Planetary Physics, University of California, Los Angeles.

Copyright 1999 by the American Geophysical Union.

Paper number 1999JA900146.

0148-0227/99/1999JA900146\$09.00
}

ships that depend on the presence of total pressure anisotropy in the medium [Hudson, 1970, 1971]:

$$
\begin{gathered}
\alpha_{\text {iso }}=\sqrt{\frac{\rho_{\Sigma}}{4 \pi}} \\
\alpha_{\text {aniso }}=\alpha_{\text {iso }} \Theta_{A},
\end{gathered}
$$

where $\Theta_{A}$ is given by

$$
\Theta_{A}=\sqrt{1-\frac{4 \pi\left(P_{\|}-P_{\perp}\right)}{B^{2}}} .
$$

This type of test has been performed in the interplanetary medium [Belcher et al., 1969; Belcher and Davis, 1971; Belcher and Solodyna, 1975; Neugebauer et al., 1984; DeKeyser et al., 1998] and at the Earth's magnetopause [Paschmann et al., 1979; Sonnerup et al., 1981]. At the magnetopause the certification of a rotational discontinuity within the current layer has long been sought as strong evidence for the Levy-PetschekSiscoe model [Levy et al., 1964] of asymmetric magnetic reconnection. While many of these tests show clear correlations in the two sides of the Walén expression of (1), the recovery of the theoretically predicted coefficient $\alpha$ has been less than satisfactory. To date, the best verification [Neugebauer et al., 1984] of the expected size even after considering absolute density calibrations is $70 \%$ of the theoretically expected size. Invariably, this number is less than theoretically expected, and for a large sample at the magnetopause the average value was 56\% [Phan et al., 1996]. Sonnerup et al. [1987, 1990] have modeled the effects of a possible acceleration of the current layer on the Walén condition test. These authors have sug- 
gested that it is the neglect of the acceleration of the free surface current layer of the rotational discontinuity (RD) that is the reason for the incomplete apparent rotation of the fluid within the observed layers at the magnetopause.

This paper examines the leverage of three systematic approaches to the ultimate certification of structures as being "Alfvénic" or sharply crested Alfvén waves (as RDs are often called). The three issues are (1) what types of measurements are available for determining the center of mass velocity $\mathbf{U}_{c m}$ required for (1); (2) if the center of mass velocity estimates were available, what systematic issues remain for testing the Walén condition with such data; and (3) can Walén be generalized for nonideal MHD for measurements across or partially through anharmonic Alfvén waves including those extreme structures usually called rotational "discontinuities."

\section{Derivation}

In a region far removed from the current-carrying layer where all particles at all random energies of all distributions perceive uniform magnetic fields during their gyro-orbits and no other spatial gradients, the individual and average bulk velocity of all species $j$ (i.e., with unique mass and charge) satisfy the same relationship

$$
c \mathbf{E}^{\infty}=-\mathbf{U}_{j}^{\infty} \times \mathbf{B}^{\infty},
$$

which restates (integrated over species) that each particle in this asymptotic region of whatever species executes the same electric drift. Averaging over an otherwise arbitrary velocity distribution of each distinguishable species gives the same relation for its average bulk velocity, $\mathbf{U}_{\perp, j}^{\infty}$ :

$$
\mathbf{U}_{\perp, j}^{\infty}=\mathbf{U}_{\perp, k}^{\infty}
$$

for distinguishable species $j$ and $k$.

Finally, in the asymptotic region the components of the center of mass velocity perpendicular to $\mathbf{B}, \mathbf{U}_{\perp, c m}^{\infty}$ are given by

$$
\mathbf{U}_{\perp, c m}^{\infty} \equiv \frac{\sum_{k} n_{k} m_{k} \mathbf{U}_{\perp, k}^{\infty}}{\sum_{l} n_{l} m_{l}}=\mathbf{U}_{\perp, j}^{\infty} \equiv \mathbf{U}_{\perp}^{\infty}
$$

Therefore, in the gradient free, asymptotic regime the center of mass of the entire plasma $\mathbf{E} \times \mathbf{B}$ drifts at the same velocity, $c\left(\mathbf{E} \times \mathbf{B} / B^{2}\right)$, as does any distinguishable fluid subspecies with labels $Z_{k}, m_{k}$. (Clearly, $U_{\|, j}^{ \pm \infty}$ need not equal $U_{\|, k}^{ \pm \infty}$, provided there are at least three distinct species in the plasma.) Thus in this asymptotic region it is also true that

$$
c \mathbf{E}^{\infty}=-\mathbf{U}_{c m}^{\infty} \times \mathbf{B}^{\infty} .
$$

As a corollary, (4) implies that the location where the traditional asymptotic Walén test is valid is between states where

$$
\mathbf{J}_{\perp}^{\infty}=0 .
$$

Equations (3) and (6a) yield two equivalent estimates of the conserved tangential electric field $\mathbf{E}_{T}^{\prime}$ in the one-dimensional current sheet's rest (primed) frame that moves with a speed $V_{\mathrm{RD}}$ along its normal $\hat{\mathbf{n}}$ :

$$
\begin{gathered}
c \mathbf{E}_{T}^{\prime}=\left(V_{\mathrm{RD}} \hat{\mathbf{n}}-\mathbf{U}_{j}^{\infty}\right) \times \mathbf{B}^{\infty} \cdot(\widetilde{I}-\hat{\mathbf{n}} \hat{\mathbf{n}}), \\
c \mathbf{E}_{T}^{\prime}=\left(V_{\mathrm{RD}} \hat{\mathbf{n}}-\mathbf{U}_{c m}^{\infty}\right) \times \mathbf{B}^{\infty} \cdot(\tilde{I}-\hat{\mathbf{n}} \hat{\mathbf{n}}),
\end{gathered}
$$

Equations (7) and (8) do not imply that the species bulk velocities are necessarily equal, since they may be satisfied by species relative drifting along $\mathbf{B}$. In the near, but asymptotic region, one-dimensional time steady current-carrying layers do require $\mathbf{J} \cdot \hat{\mathbf{n}}=0$, which then implies

$$
J_{\|}^{\infty}=0,
$$

which constrains the species slippages.

The time independence of the RD wave in the wave's rest frame that propagates along the current sheet wave normal with speed $V_{\mathrm{RD}}$ and its planar geometry imply that the vector $\mathbf{E}_{T}^{\prime}$ is conserved. This conservation law allows a connection to be made between portions of the fluid on either side of the wave train or discontinuity in the form of a vanishing jump condition,

$$
\left[\mathbf{E}_{T}^{\prime}\right]=0
$$

The null jump in the tangential electric field then implies

$$
\left[\mathbf{U}_{T, j}^{\prime}\right]=\frac{G_{j}}{B_{n}^{\prime}}\left[\frac{\mathbf{B}_{T}^{\prime}}{\rho_{j}}\right],
$$

true for any species $j$, where $B_{n}^{\prime}$ is the component of $\mathbf{B}^{\prime}$ normal to the current carrying layer and where explicit use has been made for distinguishable fluids that there is a separately conserved mass flux in the wave's rest frame along the current sheet normal given by

$$
G_{j}=\rho_{j}\left(\mathbf{U}_{j}-V_{\mathrm{RD}} \hat{\mathbf{n}}\right) \cdot \hat{\mathbf{n}} .
$$

Adding $G_{j}\left[\rho_{j}^{-1}\right] \hat{\mathbf{n}}$ to both sides of (10) yields the $j$ th distinguishable species Walén jump condition:

$$
\left[\mathbf{U}_{j}^{\prime}\right]=\frac{G_{j}}{B_{n}^{\prime}}\left[\frac{\mathbf{B}^{\prime}}{\rho_{j}}\right] \equiv \alpha_{j}\left[\frac{\mathbf{B}^{\prime}}{\rho_{j}}\right] .
$$

Because $\mathbf{B}$ is a Galilean invariant $\left[\mathbf{B}^{\prime}\right]=[\mathbf{B}]$ and $\left[\mathbf{U}^{\prime}\right] \equiv[\mathbf{U}]$, (12a) can be usefully rewritten in terms of the laboratory coordinates without first finding the RD's rest frame, namely,

$$
\left[\mathbf{U}_{j}\right]=\frac{G_{j}}{B_{n}}\left[\frac{\mathbf{B}}{\rho_{j}}\right] \equiv \alpha_{j}\left[\frac{\mathbf{B}}{\rho_{j}}\right] .
$$

A precondition for the specie Walén condition (12a) and (12b) is that individual particles of a species must pass the test that when "painted" a color on one side, they cannot be inventoried by the measurement or data evaluation process as a member of another species $j \neq k$. Even with a mass spectrometer, if there are two phase space peaks in the same species distribution observed on side 1 of the RD, they do not separately qualify, regardless of how pronounced, for their own normal mass conservation law. Only the entire group of particles in the velocity space of the $m, Z$ species satisfies the distinguishable species conservation law used in deriving (12a) and $(12 b)$.

Because the center of mass equation (8) for the asymptotic electric field has the same vectorial structure as that for a distinguishable species (7), it immediately follows that

$$
\left[\mathbf{U}_{T, \Sigma}^{\prime}\right]=\frac{G_{\Sigma}}{B_{n}}\left[\frac{\mathbf{B}_{T}}{\rho_{\Sigma}}\right],
$$

where

$$
G_{\Sigma}=\sum_{j}^{\text {all }} \rho_{j}\left(\mathbf{U}_{j}-V_{\mathrm{RD}} \hat{\mathbf{n}}\right) \cdot \hat{\mathbf{n}}
$$


is the total conserved mass flux (as viewed in the rest frame of the RD) passing across the plane of the RD. Adding $G_{\Sigma}\left[\rho_{\Sigma}^{-1}\right] \hat{\mathbf{n}}$ to both sides of (13) yields the total fluid's Walén jump condition:

$$
\left[\mathbf{U}_{\Sigma}\right]=\frac{G_{\Sigma}}{B_{n}}\left[\frac{\mathbf{B}}{\rho_{\Sigma}}\right] \equiv \alpha_{\Sigma}\left[\frac{\mathbf{B}}{\rho_{\Sigma}}\right] .
$$

From the definition of the mass flux it should be clear that $\alpha_{\Sigma}$ is defined by the phase velocity of the RD wave given by

$$
V_{\text {phase }}=\frac{B_{n}}{\sqrt{4 \pi \rho_{\Sigma}}} \Theta_{A}
$$

to yield the expression

$$
\alpha_{\Sigma}=\frac{\rho_{\Sigma} V_{\text {phase }}}{B_{n}}=\Theta_{A} \sqrt{\frac{\rho_{\Sigma}}{4 \pi}}=\alpha_{\text {aniso }}
$$

To use observations to evaluate $\alpha_{\Sigma}$ in (15a) requires mass separation and model independent determination of the required moments of the intrinsically three dimensional distribution functions of all species. Collisionless arguments would argue that there are portions of velocity space on one side of the RD that do not connect across the layer but are reflected by it [Cowley, 1980]. Such reflections place accessibility constraints on the forms of the distribution function on both sides of the discontinuity that have been detected at the magnetopause but overlooked in the data reduction assumptions at interplanetary RDs. These circumstances limit in an unknown way the precision of unpacking the count rate distributions under this hypothesis from particle spectrometers that separate species only by energy per unit charge, $E / Z$. This implies that fitting to preassigned functional forms to peaks in the observed count rate distribution rather than direct model independent numerical integration of a mass per charge resolved plasmas may be a source of significant quantitative error. Stated positively, the act of measurement of ion species with an $E / Z$ detector is an entropy-producing process that cannot be inverted in a model independent way. Hence it follows that ion moments from an $E / Z$ detector cannot be expected to be quantitatively precise for use in either (12a) and (12b) or the related version (15a)-(15c) for the mass density weighted sum $\mathbf{U}_{\Sigma}$. When the separate species are not discernible in the $E / Z$ measurements as in the hot magnetosheath, there are additional sources of ambiguity for the data interpretation. P. PuhlQuinn and J. D. Scudder (manuscript in preparation, 1999) illustrate that the systematics of not being able to sort by mass imply for typical parameters that the Walén slope with ions would be inferred to be systematically too small relative to theory from this effect alone. These results support observations, given the fact that previous researchers and we show (Figures 3-6, and 10) that the ion slope is observationally too small.

By contrast, the ambient astrophysical plasmas of interest contain only electrons as anions. The observations of the negatively charged constituents in the plasma by $E / Z$ techniques constitute de facto an anion mass spectrometer; there are, however, two classes of electrons: ambient electrons of the medium and photoelectrons made at the spacecraft surface and largely confined there by the spacecraft's floating potential. Provided the photoelectrons can be accurately removed, the electron fluid represents an ideal vehicle to test (12a) and (12b) at the distinguishable species level for the rotational discontinuity jump conditions via (12a) and (12b) with $j=e$, $\rho_{e}=m_{e} n_{e}$, and $G_{e}=m_{e} n_{e}\left(\mathbf{U}_{e}-V_{\mathrm{RD}} \hat{\mathbf{n}}\right) \cdot \hat{\mathbf{n}}$. Specialized in this form, (12a) and (12b) become

$\left[\mathbf{U}_{e}^{\infty}\right]=\frac{n_{e}\left(\mathbf{U}_{e}-V_{\mathrm{RD}} \hat{\mathbf{n}}\right) \cdot \hat{\mathbf{n}}}{B_{n}}\left[\frac{\mathbf{B}^{\infty}}{n_{e}^{\infty}}\right]=\frac{G_{e}}{m_{e} B_{n}}\left[\frac{\mathbf{B}^{\infty}}{n_{e}^{\infty}}\right]$.

\section{What Value of $G_{e} / m_{e}$ is Expected?}

The size of the electron Walén proportionality constant in (16) deserves evaluation in two limits: (1) an electron-proton plasma and (2) an electron-proton-helium plasma. In the first category the steady state requirement of zero current along the current sheet normal in the quasi-neutral approximation greatly simplifies the evaluation of $G_{e}$ in terms of $G_{\Sigma}$. In the second case, multiple ions imply that it is possible that neither ion specie is moving in the asymptotic regime at the same speed as the electrons along the magnetic field. Furthermore, the minor ion could be either singly or multiply charged, depending on its origin. We will estimate below the size of $G_{e}$ in the $\mathrm{He}$ minor ion limit with charge $Z_{\mathrm{He}}$ and in the limit that the slippage between the ions is less than the electron flow speed along the normal.

Case (1), for the electron-proton plasma, the zero-current condition along the RD normal guarantees that their normal charge fluxes are equal, while quasi-neutrality guarantees equal numbers of protons and electrons, so that in case (1)

$$
U_{e, n}^{\prime}=U_{p, n}^{\prime}=U_{c m, n}^{\prime}
$$

so that

$$
\frac{G_{e}}{m_{e}} \equiv \frac{G_{\Sigma}}{m_{p}+m_{e}}
$$

Case (2), quasi-neutrality for helium in charge state $Z_{\mathrm{He}}$, proton, and electron plasma mixture implies that the helium number density is given by

$$
n_{\mathrm{He}}=\frac{n_{e}-n_{p}}{Z_{\mathrm{He}}} .
$$

Ancillary equations that assist the multicomponent plasma are the conditions of zero current along the normal

$$
\sum_{j}^{\text {ion }} n_{j} Z_{j}\left(\mathbf{U}_{j}-V_{\mathrm{RD}} \hat{\mathbf{n}}\right) \cdot \hat{\mathbf{n}}=n_{e}\left(\mathbf{U}_{e}-V_{\mathrm{RD}} \hat{\mathbf{n}}\right) \cdot \hat{\mathbf{n}},
$$

coupled with quasi-neutrality

$$
\sum_{j}^{\text {ion }} n_{j} Z_{j}=n_{e}
$$

that together imply that

$$
\sum_{j}^{\text {ion }} n_{j} Z_{j} \mathbf{U}_{j} \cdot \hat{\mathbf{n}}=n_{e} \mathbf{U}_{e} \cdot \hat{\mathbf{n}} .
$$

Assume that the helium speed along the normal does not match the protons, so that there is an allowed dimensionless differential motion $\varepsilon$ given by

$$
\varepsilon=\frac{\mathbf{U}_{\mathrm{He}} \cdot \hat{\mathbf{n}}-\mathbf{U}_{p} \cdot \hat{\mathbf{n}}}{\mathbf{U}_{e} \cdot \hat{\mathbf{n}}} .
$$


Define $\Delta$ as the fractional departure of the number density of electrons and protons relative to protons by

$$
\Delta \equiv \frac{n_{e}-n_{p}}{n_{p}} \equiv \frac{Z_{\mathrm{He}} n_{\mathrm{He}}}{n_{p}}
$$

then

$$
\frac{n_{e}-n_{p}}{n_{e}}=\frac{\Delta}{\Delta+1},
$$

and the total mass flux constant consistent with zero current along the normal and quasi-neutrality is given by the exact expression:

$G_{\Sigma} \equiv \frac{\mu G_{e}}{m_{e}}\left[\frac{1}{1+\Delta}+\frac{\Delta \varepsilon}{(1+\Delta)^{2}} \frac{4-Z_{\mathrm{He}}}{Z_{\mathrm{He}}+4 \Delta}+\frac{m_{e}}{\mu}\right]$,

where we have used the reduced mass definition

$$
\mu=m_{p}\left(1+4 \frac{\Delta}{Z_{\mathrm{He}}}\right)
$$

to obtain the approximate result (first order in $\Delta$ ):

$$
\frac{G_{e}}{m_{e}} \simeq \frac{G_{\Sigma}}{\mu}\left\{1-\Delta\left[\frac{\varepsilon}{Z_{\mathrm{He}}}\left(4-Z_{\mathrm{He}}\right)-1\right]\right\} .
$$

Thus in both cases we may summarize that

$$
\frac{G_{e}}{m_{e}}=n_{p} U_{c m, n}^{\prime}\left\{1-\Delta\left[\frac{\varepsilon}{Z_{\mathrm{He}}}\left(4-Z_{\mathrm{He}}\right)-1\right]\right\} .
$$

Putting the general Alfvén phase velocity relation

$$
U_{c m, n}^{\prime}= \pm \frac{B_{n}}{\sqrt{4 \pi \mu n_{p}}}\left[1-\frac{4 \pi\left(P_{\|}-P_{\perp}\right)}{B^{\prime 2}}\right]^{1 / 2}
$$

into (29) yields the desired simplification for $G_{e} / m_{e}$ :

$$
\begin{aligned}
\frac{G_{e}}{m_{e}}= & \pm \frac{B_{n}}{1+\Delta\left[\frac{\varepsilon}{Z_{\mathrm{He}}}\left(4-Z_{\mathrm{He}}\right)-1\right]} \\
& \cdot \sqrt{\frac{n_{p}}{4 \pi \mu}\left[1-\frac{4 \pi\left(P_{\|}-P_{\perp}\right)}{B^{\prime 2}}\right]} .
\end{aligned}
$$

In this way the multifluid expectation for the electron asymptotic Walén condition has the form:

$$
\left[\mathbf{U}_{e}^{\infty}\right]= \pm \frac{\sqrt{\frac{\rho_{\Sigma}}{4 \pi}\left[1-\frac{4 \pi\left(P_{\|}-P_{\perp}\right)}{B^{\prime 2}}\right]}}{1+\Delta\left[\frac{\varepsilon}{Z_{\mathrm{He}}}\left(4-Z_{\mathrm{He}}\right)-1\right]}\left[\frac{\mathbf{B}^{\infty}}{\mu n_{e}^{\infty}}\right] .
$$

Comparison of (32) with (1) and (2b) clearly indicates that the form for the anisotropic electron Walén jump conditions is almost exactly the same as that for the asymptotic ion jump forms and the center of mass form, namely,

$$
\left[\mathbf{U}_{e}^{\infty}\right]= \pm \alpha_{\text {aniso }}^{e}\left[\frac{\mathbf{B}^{\infty}}{\rho_{\Sigma}}\right]
$$

with

$$
\alpha_{\text {aniso }}^{e} \equiv R_{A}^{e} \alpha_{\text {aniso }},
$$

where the Alfvén ratio for electrons $R_{A}^{e}$ is given by the approximate expression

$$
R_{A}^{e} \simeq\left\{1-\Delta\left[\frac{\varepsilon}{Z_{\mathrm{He}}}\left(4-Z_{\mathrm{He}}\right)-1\right]\right\} .
$$

Specializing (33b) to the three cases discussed above recovers with $\varepsilon<1$ :

$$
\begin{gathered}
R_{A}^{e}(\Delta=0)=1 \\
R_{A}^{e}\left(Z_{\mathrm{He}}=2\right) \simeq[1-\Delta(\varepsilon-1)] \\
R_{A}^{e}\left(Z_{\mathrm{He}}=1\right) \simeq[1-\Delta(3 \varepsilon-1)] .
\end{gathered}
$$

The form of the electron asymptotic Walén condition given in (33a) and (33b) has been derived for jumps (brackets) that connect gradient free, $\mathbf{J}=0$, "asymptotic" regimes. We now move to relax the asymptotic comparison requirement so that measurements that are undoubtedly contained within the layer may be included in the regression analysis that empirically ascertains the proportionality constant $\alpha_{e}$ of the Walén relation (33b) that allows evaluation of $R_{A}^{e}$. We retain the assumption that the time independent wave frame exists but relax the need to compare data points that are only certified to be outside the field rotation region.

\section{Generalized Walén Condition}

As the time resolution of plasma detectors improves and as experimenters use denser samplings to overdetermine the Walén condition proportionality constant of (12, 15, 33(a), and 33(b)), it is almost unavoidable that data will be acquired within the current-carrying layer that is implementing the twist of the magnetic field and that is shearing and accelerating the plasma. To some extent, this is also a factor in the Walén and deHoffmann-Teller tests [e.g., Sonnerup et al., 1981; Aggson et al., 1983] that have already been performed at the magnetopause and in the interplanetary medium using long stretches of Alfvénic wave trains [Belcher et al., 1969; Belcher and Davis, 1971; Belcher and Solodyna, 1975]. In such a current-carrying region the electric field $\mathbf{E}$ is no longer simply related to the center of mass motion as in (6a), since the Hall and ambipolar electric fields will be important in this region.

For a steady one-dimensional rotational discontinuity, Maxwell's equations guarantee that the tangential electric field is a true constant vector throughout the currents inside the layer. Maxwell does not, however, guarantee that the electric field may be inferred within the current-carrying layer with the form

$$
c \mathbf{E}(\mathbf{x}) \neq-\mathbf{U}_{c m}(\mathbf{x}) \times \mathbf{B}(\mathbf{x})
$$

obtained by dropping the superscript $\infty$ in (6a), since (34) is just the first term in the generalized Ohm's law [Rossi and Olbert, 1970] that can only be written approximately and then has the form

$$
\begin{aligned}
\mathbf{E}(\mathbf{x}) \simeq & -\frac{\mathbf{U}_{c m}(\mathbf{x}) \times \mathbf{B}(\mathbf{x})}{c}+\frac{\mathbf{J}(\mathbf{x}) \times \mathbf{B}(\mathbf{x})}{e c n_{e}(\mathbf{x})}-\frac{\nabla \cdot \mathbf{P}_{e}(\mathbf{x})}{e n_{e}(\mathbf{x})} \\
& -\frac{m_{e}}{\mu} \frac{\nabla \cdot \sum_{\text {ions }} \mathbf{P}_{i}(\mathbf{x})}{e n_{p}(\mathbf{x})}+\frac{m_{e}}{n(\mathbf{x}) e^{2}} \\
& \cdot\left\{\frac{\partial \mathbf{J}(\mathbf{x})}{\partial t}+\nabla \cdot\left[\mathbf{U}_{c m}(\mathbf{x}) \mathbf{J}(\mathbf{x})+\mathbf{J}(\mathbf{x}) \mathbf{U}_{c m}(\mathbf{x})\right]\right\} \\
& +\eta(\mathbf{x}) \cdot \mathbf{J}(\mathbf{x}),
\end{aligned}
$$


where $\mu n_{p} \equiv \sum n_{j} m_{j}$. The leading order corrections in (35) to the incorrect surmisal of (34) occur in the presence of crossfield currents and pressure gradients. An Alfvén wave surely has cross-field currents.

An exact (rather than approximate) form for the electric field is obtained from inverting the electron momentum equation:

$$
\mathbf{E}_{e}(\mathbf{x})=\mathbf{E}_{M, e}(\mathbf{x})+\mathbf{E}_{A, e}(\mathbf{x})+\mathbf{E}_{I, e}(\mathbf{x})+\mathbf{E}_{C, e}(\mathbf{x}),
$$

where the electron form of the motional electric field $\mathbf{E}_{M, e}$ is given by

$$
\mathbf{E}_{M, e}(\mathbf{x})=-\frac{\mathbf{U}_{e}(x) \times \mathbf{B}(x)}{c}
$$

the electron form of the ambipolar electric field $\mathbf{E}_{A, e}$ is given by

$$
\mathbf{E}_{A, e}(\mathbf{x})=-\frac{\nabla \cdot \mathbf{P}_{e}(\mathbf{x})}{e n_{e}(\mathbf{x})}
$$

the electron form of the inertial electric field $\mathbf{E}_{I, e}$ contributions are given by

$$
\begin{aligned}
\mathbf{E}_{I, e}(\mathbf{x})= & -\frac{m_{e}}{e n_{e}(\mathbf{x})}\left\{\frac{\partial n_{e}(\mathbf{x}) \mathbf{U}_{\mathbf{e}}(\mathbf{x})}{\partial t}+\nabla \cdot\left[n_{e}(\mathbf{x}) \mathbf{U}_{\mathbf{e}}(\mathbf{x}) \mathbf{U}_{\mathbf{e}}(\mathbf{x})\right]\right\} \\
& -\frac{G M_{o} m_{e}}{e r^{2}} \hat{\mathbf{r}}
\end{aligned}
$$

and the electron form of the collisional electric field $\mathbf{E}_{C, e}$ is denoted by

$$
\mathbf{E}_{C, e}(\mathbf{x})=+\frac{1}{e n_{e}(\mathbf{x})}<\left.\frac{\delta f(\mathbf{x})}{\delta t}\right|_{c} m_{e} \mathbf{v}>\mathbf{v}_{\mathbf{e}} .
$$

It is central to the purposes of this paper to note that (36)(40) yield expressions for the electric field that are exact, spatially dependent, and contain no reference to the number of ion species in the plasma nor their slippages with respect to one another. This situation should be contrasted with (35), which requires simultaneous knowledge of the different concentrations, flow velocities, and pressures of all the species in the plasma.

The absence of the $\mathbf{J} \times \mathbf{B}$ term in the electron expression for E (36)-(40) is not an omission nor an approximation, since (36) is exact; using the first two terms of (35), one readily sees that the center of mass form of the generalized Ohm's law approximates (if clumsily) $\mathbf{E}_{M, e}$ when the first two terms of (35) are retained. This awkward reconstruction of (36) from (35) seems to imply that the terms neglected involving ion slippages and masses detract from the precision of $\mathbf{E} \simeq \mathbf{E}_{M, e}$. Equation (36) makes clear that this is not the correct interpretation but that the center of mass form for $\mathbf{E}$ is awkwardly accommodating the clear-cut implications of the electron momentum equation. If we accept the formal ordering of the terms in (36), it strongly suggests (as is well known [Rossi and Olbert, 1970; Scudder, 1997]) that the magnetic field is convected with the spatially varying electron bulk velocity, with corrections from the ambipolar, inertial, and collisional terms. This, in turn, implies that the conserved tangential electric field guaranteed by Maxwell can be at the same level of precision determined from the local components of $\mathbf{E}_{M, e}$ transverse to the RD normal.

Because (37) locally has the same structure as (6a), it im- mediately follows that the form of the electron Walén test (33), is also valid all the way through the current-carrying layer of the wave train or resolved $\mathrm{RD}$, provided the scales of the layer do not become so small that the ambipolar correction, $\mathbf{E}_{A, e}$, is a significant contributor to $\mathbf{E}_{T}(x)$.

We may therefore state that the electron or generalized Walén test for the observer wandering outside and in the current-carrying layer of the wave train or resolved discontinuity layer, with or without traversing, is

$$
\begin{aligned}
{\left[\mathbf{U}_{e}(x)\right] } & =\frac{G_{e}}{m_{e} B_{n}}\left[\frac{\mathbf{B}(x)}{n_{e}(x)}\right] \\
& =R_{A}^{e} \alpha_{\Sigma}\left[\frac{\mathbf{B}(x)}{\mu n_{e}(x)}\right], \frac{\left|\mathbf{E}_{A, e} \cdot(I-\hat{\mathbf{n}} \hat{\mathbf{n}})\right|}{\left|\mathbf{E}_{M, e} \cdot(I-\hat{\mathbf{n}} \hat{\mathbf{n}})\right|} \ll 1
\end{aligned}
$$

where the proportionality constant is the same as in (33). We now introduce the notation $[\eta(\mathbf{x})]$ to denote differences between any two points in the disturbance and contrast it with $[\eta]$ as used in (1), which denotes jumps between asymptotic, current free regions on either side of the wave train or currentcarrying layer.

Finally, it should be mentioned that there is an analogous form to (36) for the electric field specific to each identifiable fluid involved in the plasma. By changing $e \rightarrow-Z_{i} e$ and subscripts $(e \rightarrow i)$, these forms can be found from (36)-(40). These forms are not, however, of much use for our purposes, since an accurate determination with them of $\mathbf{E}_{T}$ within current-carrying layers with ion gyroscales of necessity has important lowest-order contributions from the divergence of the nongyrotropic ion pressure tensor term as well as the ion dynamic pressure inertial term [Parker, 1957]. Accordingly, the argument of the previous paragraph cannot be carried through by analogy to obtain another Walén form relation between distinct ion components within the layer (see (43b)).

\section{Alternate View of Ion Walén Relation}

By definition, the (single-species ion) form of the ion velocity spatial profile through the stationary wave would be given in the wave frame by the relationship

$$
\mathbf{U}_{i}^{\prime}(\mathbf{x})=\mathbf{U}_{e}^{\prime}(\mathbf{x})+\frac{\mathbf{J}(\mathbf{x})}{e n_{e}(\mathbf{x})},
$$

which is also true for the laboratory coordinates:

$$
\mathbf{U}_{i}(\mathbf{x})=\mathbf{U}_{e}(\mathbf{x})+\frac{\mathbf{J}(\mathbf{x})}{e n_{e}(\mathbf{x})} .
$$

Since Alfvénic structures are implemented with currents, the distinction between the ion spatial profile and the electrons must be significant, since it is the current through the Lorentz force that causes the ion center of mass to undergo transverse undulations.

Using a first integral of the electron form of the Walén relation (41) and Ampére's equation in a one-dimensional steady state current layer yields

$$
\begin{aligned}
\mathbf{U}_{i}(\mathbf{x})= & \mathbf{U}_{e}\left(\mathbf{x}_{0}\right) \pm \alpha_{e}\left[\frac{\mathbf{B}(\mathbf{x})}{\rho_{\Sigma}(\mathbf{x})}-\frac{\mathbf{B}\left(\mathbf{x}_{0}\right)}{\rho_{\Sigma}\left(\mathbf{x}_{0}\right)}\right] \\
& +\frac{c}{4 \pi e n_{e}(\mathbf{x})} \nabla \times \mathbf{B}(\mathbf{x}) .
\end{aligned}
$$

This relation has a jump form 


$$
\left[\mathbf{U}_{i}(\mathbf{x})\right]= \pm \alpha_{e}\left[\frac{\mathbf{B}(\mathbf{x})}{\rho_{\Sigma}(\mathbf{x})}\right]+\left[\frac{c}{4 \pi e n_{e}(\mathbf{x})} \nabla \times \mathbf{B}(\mathbf{x})\right]
$$

Under the stated assumptions, (43a) and (43b) are exact. Note that in form the ion expression (43b) differs functionally from that for the electrons (41). Finally, the form (43b) was not previously used when the Walén test was undertaken, since the tacit assumption was made that (1) was appropriate for the ions. Clearly, (43b) could be generalized to include additional ion species with different charges. Such generalizations would represent further structural changes in the expected ion flow variations. Only in the monochromatic idealizations of textbooks can a long-wavelength wave not have potentially measurable corrections to the ion flow velocity from the Lorentz term of (43b). The multiple Fourier environment of the Alfvénic disturbances seen in nature and modeled by hybrid simulations [Krauss-Varban et al., 1995] implies that the spatial profiles of $\mathbf{U}_{i}(x)$ have important corrections to the center of mass expectations of the Walén form. The electron form of the Walén conditions (43) is expected to be satisfied, regardless of the spectral content of the resolved waveform, so long as the spatial wavenumbers are well above the electron inertial length.

\section{Examples}

Recently, there has been the opportunity with the Global Geospace Science (GGS) Polar Hydra, Magnetic Field Experiment (MFE), and Electric Field Investigation (EFI) instruments to sample current-carrying layers near the magnetopause and test them for possible rotational character, long taken to be the most sensible evidence for a locally open magnetosphere. The three-dimensional measurements of the plasma electrons and ions between $5 \mathrm{eV} / q$ and $20 \mathrm{keV} / q$ were performed by the Hydra instruments in the "default" mode as described fully by Scudder et al. [1995]; the magnetic field measurements were provided by the MFE instrument as described by Russell et al. [1995]. Spacecraft floating potentials have been determined after extensive collaborations with the electric field team and the data of the EFI experiment [Harvey et al., 1995] concerning the variation of the floating potential and Hydra's perceived variation of the plasma return current that accompanies those variations [Scudder et al., 1999]. The plasma moments of electrons and ions are determined from Hydra data with a 13.8 s cadence based on ion and electron energy sweeps on 12 separate detectors stepped simultaneously across the entire energy range in $1.15 \mathrm{~s}$ with ion sweeps interleaved between each of six electron sweeps, while the routine MFE records contain data of the vectorial magnetic field components at $8 \mathrm{~Hz}$. Within Hydra's measurement stream, onboard information of the magnetic field is available at $54 \mathrm{~Hz}$, so that the temporal resolution of the MFE telemetry stream can be augmented, especially insofar as angular information is concerned. The $54 \mathrm{~Hz}$ magnetic data and the $8 \mathrm{~Hz}$ data have been cross calibrated and anomalies documented and removed from both data streams.

An example of a candidate RD current layer encountered by Polar is illustrated in Figure 1, where electron density, magnetic intensity, and three despun payload Cartesian components of $\mathbf{B}$ are shown. The magnetic field traces are at a resolution of $54 \mathrm{~Hz}$, the wide histogram-like steps across all panels of Figure 1 reflect the time-aliasing interval of $13.8 \mathrm{~s}$ of Hydra moment quantities, and the shorter steps in the density panel reflect the temporal duration and $1.15 \mathrm{~s}$ resolution of the electron energy sweep subcycle measurements (which are interspersed with the ions during the 13.8 s cycle). The numbers affixed to the wide histogram steps denote indices of those moment subintervals used in the Walén analysis of this particular interval (the selection of which is described later in this section). The histogram steps in the magnetic field panels represent the $13.8 \mathrm{~s}$ average (solid lines) and variance (dashdotted lines) of the $54 \mathrm{~Hz}$ data. It is this average and variance that are used when determining $\alpha_{e}$ in (41) since they represent $\mathbf{B}$ on the same timescale as the moment-determined flow velocity. Although $13.8 \mathrm{~s}$ is required to determine the vectorial and tensorial moments, the density and temperature of the electrons are available at $1.15 \mathrm{~s}$ (as mentioned above) to help with the time-aliasing issues and are plotted beneath the histogram of $13.8 \mathrm{~s}$ densities determined from full numerical integration of the three-dimensional phase space. Prior to numerical integration, electron and ion measurements have been shifted in energy to correct for the known floating potential of the spacecraft (provided when available by EFI or from iteration against the spacecraft return current-potential calibration curve [Scudder et al., 1999]).

The $54 \mathrm{~Hz}$ magnetic field data (available within Hydra's telemetry allocation) for each flagged interval was used in a minimum variance analysis determination of $\hat{\mathbf{n}}$, the boundary normal direction. Determining $\hat{\mathbf{n}}$ serves two main purposes in relation to the Walén tests: (1) it allows a check on whether $B_{n}$ is nonzero across the boundary (a requirement for normal mass flux) and whether $B_{n}$ is a constant (a requirement from Maxwell's equations) and (2) knowing $\hat{\mathbf{n}}$, the sign of $B_{n}$ and the sign of $\alpha$ (the Walén fit slope) allows an assignment of geophysical direction to the mass flux across the boundary (briefly discussed later). Figure 2 illustrates the magnetic field in boundary normal coordinates $\left(B_{n}, B_{T 1}\right.$, and $\left.B_{T 2}\right)$ for the same interval as in Figure 1. Notice that for this particular example, $B_{n}$ is $\sim-11 \pm 9 \mathrm{nT}$. Also of interest is that the interval (unmarked, but would have been numbered 89) with the largest departures from this average value were excluded in the automatic minimization procedure discussed below.

Relationship (41) suggests using all possible component pairs of Cartesian differences of $U_{e, j}^{\mathrm{lab}}$ and $B_{j}$ to test for the suggested linear correlation and the size of the slope. For example, the interval illustrated in Figure 1 spans five moment subintervals (only four of which are labeled, for reasons stated below). Taking all possible pairings of these five subintervals (where order does not matter) yields $5 ! /[(5-2) ! 2 !]=10$ pairings, from which 10 unique vectorial differences in $\mathbf{U}_{e}^{\mathrm{lab}}(x)$ and $\mathbf{B}^{\text {lab }}(x) / \rho_{\Sigma}(x)$ can be constructed. This translates to 30 unique scalar differences $(10 \times 3$ components $)$, which are all simultaneously used to interrogate the relationship described by (41). It was found for the interval in Figure 1 that all possible pairings of the four moment subintervals labeled 87, 88, 90, and 91 satisfied the electron Walén relationship described by (41) within the uncertainties described in the next paragraph.

An acceptable fit for an RD in the context of MHD must both have (1) an acceptably ranked linear correlation that describes all Cartesian components of the vector equation and (2) the correct size of $\alpha$ as determined by the density and possibly the anisotropic total $\beta$ of the plasma using (2b) and (33e). Since the correlation sought is between derived parameters that both have errors, the slope has been determined by using the method of hypothesis testing that incorporates errors for the $B_{j}$ and $U_{k, j}$ simultaneously. The high-resolution magnetometer data are averaged to correspond to the $13.8 \mathrm{~s}$ alias- 

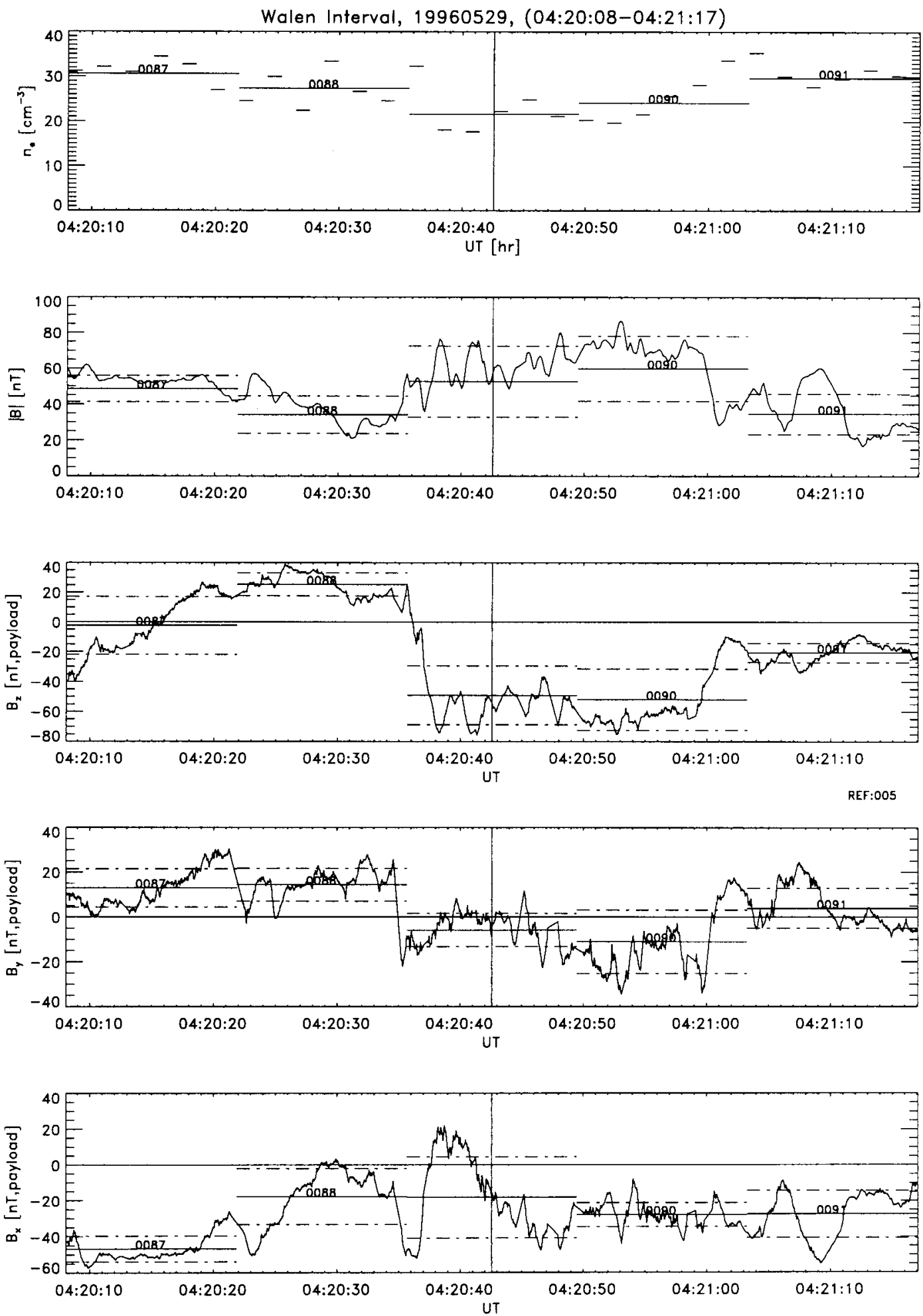

Figure 1. Hydra electron density and (MFE) magnetic field components.

ing interval of the moment quantities, and the component variances $\sigma_{B_{j}}$ of these average quantities are used for the uncertainties of the average components of $B_{j}$ (see Figure 1). Uncertainties of $\rho_{\Sigma}$ follow from Poisson statistics or the variability of the $1.15 \mathrm{~s}$ density estimates from the energy subsweeps within the $13.8 \mathrm{~s}$ moment cycle time, whichever is larger. From a previous study of the precision of the determination of the electron bulk velocity [Scudder et al., 1986], it is known that the rms error for each Cartesian component of the electron bulk velocity for this instrument scales with the electron temperature $T_{e}$ such as

$$
\sigma_{U_{e, j}}=25 \mathrm{~km} / \mathrm{s}\left(\frac{T_{e}}{2.0 \times 10^{5} \mathrm{~K}}\right)^{1 / 2} .
$$

At first glance, this error would seem to be large, especially as judged against that for ions. However, the thermal width for a 

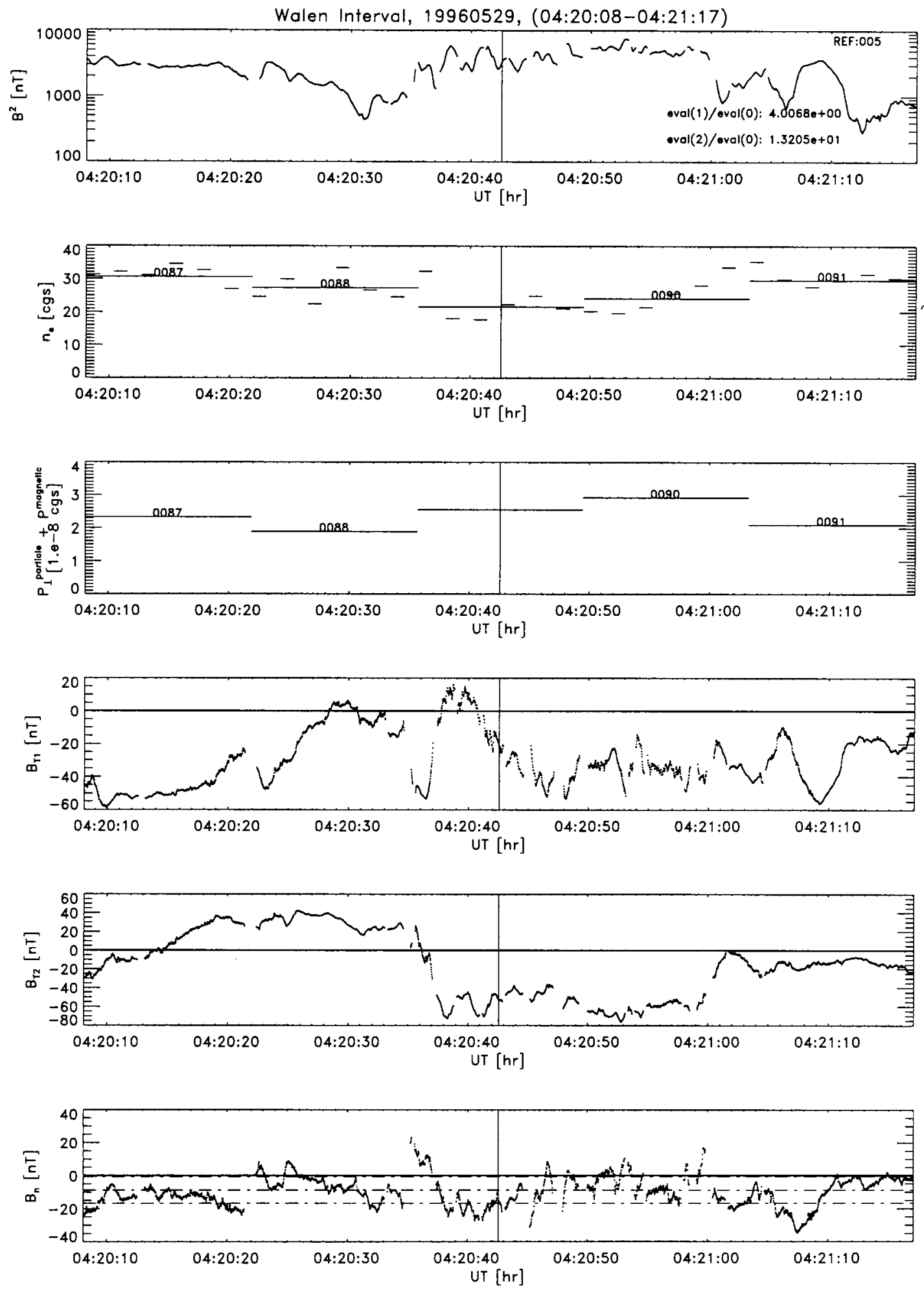

Figure 2. Magnetic Field Experiment (MFE) $B$ field in boundary normal coordinates.

$20 \mathrm{eV}$ electron distribution function is in excess of $2650 \mathrm{~km} / \mathrm{s}$. The precision statement of (44) corresponds to finding the centroid of a distribution of $e$-folding full width of $5300 \mathrm{~km}$ at a precision of $\frac{1}{2} \%$. The in-flight determination of (44) has also been substantiated by model studies with known distributions with Hydra class sampling strategies and are also substantiated using three axis $\mathbf{E}$ and $\mathbf{B}$ measurements in section 8.
We solve the hypothesis testing problem as a variant of the problem described by Press et al. [1992] for the determination of the best fit line that simultaneously organizes all Cartesian components as a model for coordinate pairs each with a predetermined error. We have modified this approach to constrain the line of best fit to have zero intercept and determined the size and $68 \%$ confidence error bound of the 

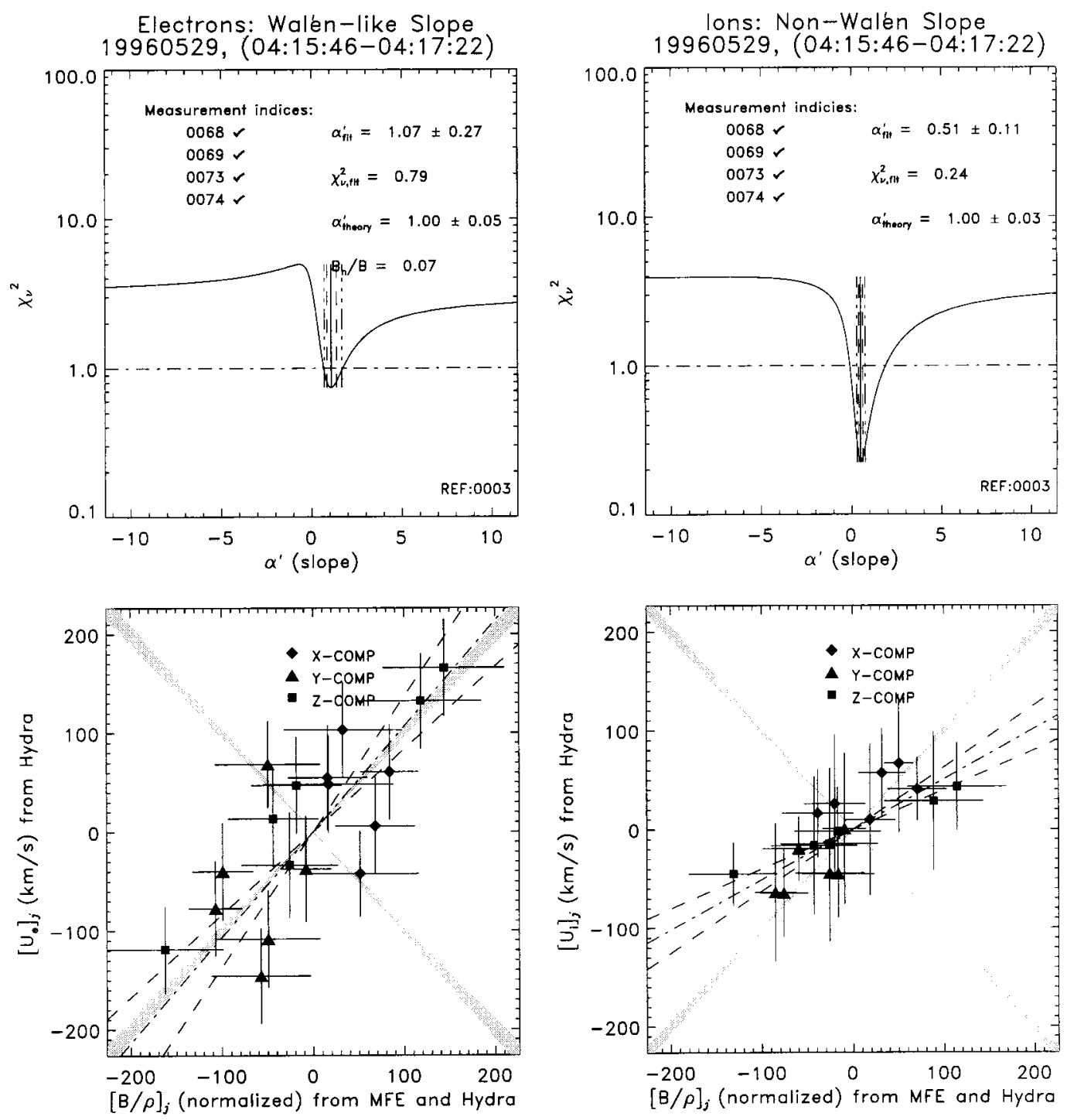

Figure 3. Walén fit $\chi^{2}$ surface, fit with error bounds for $\alpha_{e} \simeq 1$.

best fit slope $\alpha$ of (41) that describes the observed coordinate pairs.

Two plots for RDs at well separated time intervals are illustrated in Figures 3 and 4. Figures 3 and 4, left, pertain to the electron Walén test results for the indicated time interval using (41); Figures 3 and 4, right, contain the results of the Walén test (1) using the Hydra ion measurements during the same time interval as the electrons, assuming, as have previous workers, that all ion counts were made by protons. Each plot displays the data points in the ([ $\left.\left.U_{k, j}^{\mathrm{lab}}\right],\left[B_{j} / \rho\right]\right)$ space (Figures 3 and 4 , bottom) for all components $j$ of the $k$ th species and variation of reduced chi-square, $\chi_{\nu}^{2}(\alpha)$, as the fit slope is varied (Figures 3 and 4, top). Data pairs of the different Cartesian components have different symbols. Notice that the $\left[B_{j} / \rho\right]$ data points have been normalized by $\left|\alpha_{\text {theory }}^{-1}(\Delta=0)\right|$, so that the expected slope $\alpha^{\prime}$ of a "successful" Walén test would be \pm 1 . The best fit selected line is indicated by the dash-dotted line, and its $68 \%$ confidence bounds are denoted by the dashed lines on either side. The shaded arms that comprise each line of the "X" are the predictions for the line using (41) and the observed variance of $\alpha$ as determined by the measured total plasma mass density and pressure anisotropy. These line wedges reflect the dispersion in the expected scaled slope $\alpha^{\prime} \equiv$ $\sigma_{\alpha} /\langle\alpha\rangle_{x}$, consistent with the observed variation of the theoretically required "constant," $\langle\alpha\rangle_{x}$, determined from the spatial average of the electron and ion measurements of Hydra including total pressure anisotropy through the same space-time interval used for the fit. The two arms of the $\mathrm{X}$ wedges reflect the ambiguity of the sign in (1). This sign denotes the relative flow direction in the rest frame of the RD of the flow along the normal, $U_{n}^{\prime}$, and the component of $\mathbf{B}^{\prime}$ along that normal, $B_{n}^{\prime}$. This sign plays a crucial role in the assessment of the location of the observer relative to a possible separator. If the RD were standing in inertial space, then the sign of $\alpha$ denotes the direction of the mass flux through the plane of the RD relative to $B_{n}^{\prime}$. In the particular examples of Figures 3 and $4, \alpha^{\prime}$ is positive, indicative of flow in the RD rest frame, $U_{e, n}^{\prime} \hat{\mathbf{n}}$, being along the same direction as $B_{n}^{\prime} \hat{\mathbf{n}}$.

By contrast, Figures 3 and 4, right, illustrate Hydra ion velocity measurements in the same format with the same normalization; these ion moment quantities are determined by numerical integration using ion energy and angular sweeps interleaved with the electron information used in Figures 3 and 4, left. These columns in Figures 3 and 4 illustrate the good 

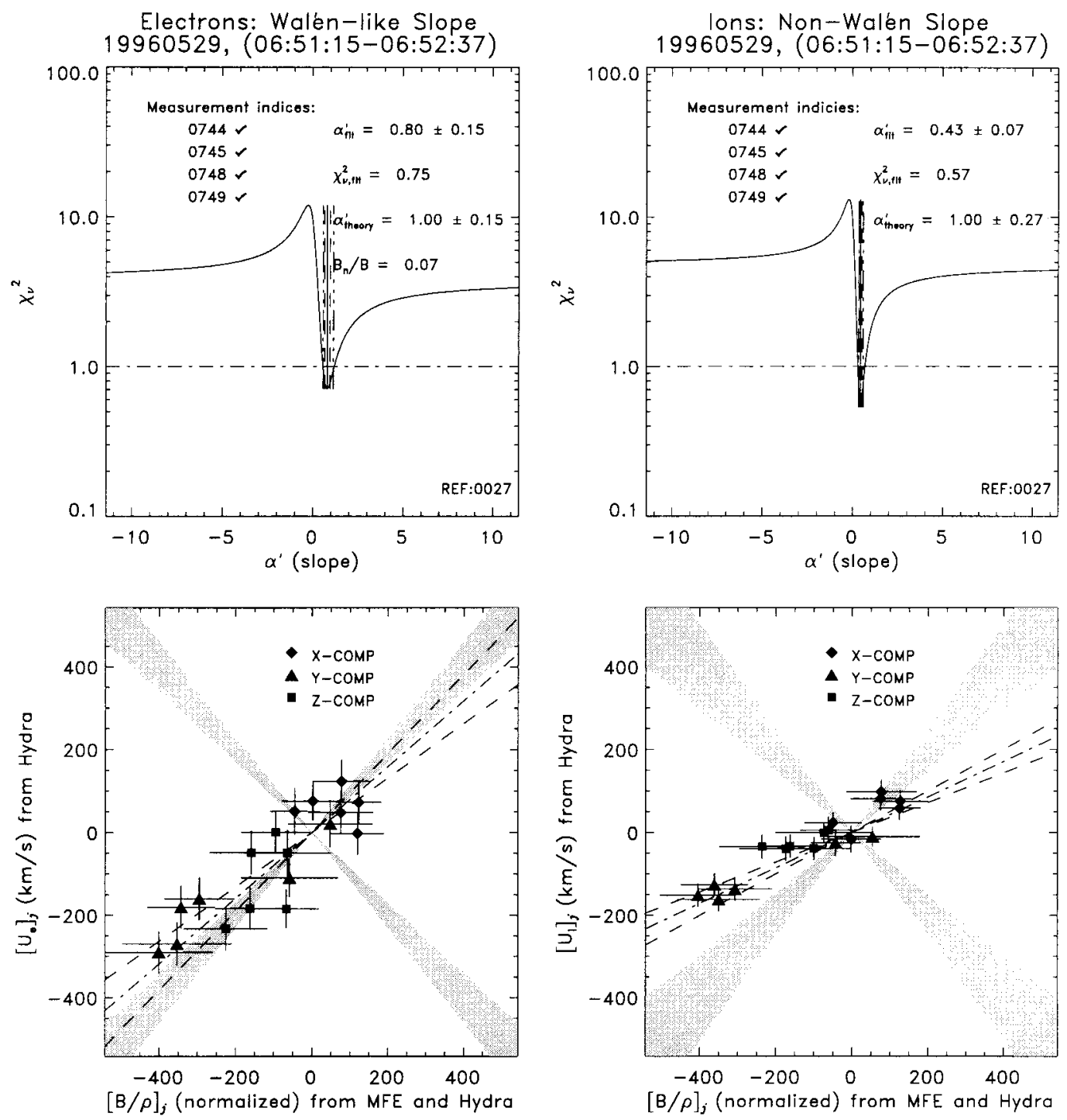

Figure 4. Additional Walén fit $\chi^{2}$ surface, fit with error bounds for $\alpha_{e} \simeq 1$.

correlation of the $\mathbf{U}_{i}$ and $\mathbf{B}$ changes, but with decidedly incorrect slopes. The ion slope is smaller than that of the electrons and definitely less than unity, with $\alpha_{i}^{\prime} \simeq 0.5 \pm 0.1$ of the expected value, but similar to the previous reports of this type of regression analysis.

Figure 5 illustrates another interval in the same format when a successful electron Walén test with $\alpha_{e}^{\prime} \simeq-1$ was traversed, indicating that the mass flux in the rest frame of the RD was opposite in relationship to $B_{n}$ from the example in Figures 3 and 4. Again, high-quality agreement is shown, with normalized $\chi_{\nu}^{2}$ that is small and acceptably ranked with a near-unit slope and an error bar that overlaps that expected from the Hydra ion and electron pressure anisotropy and density data. As in Figures 3 and 4, the accompanying ion flow velocity measurements shown in Figure 5, right, do not support the expected proportionality, even though the ion and field changes are linearly correlated. With a dimensionless slope of -0.23 the ion shears do not meet the stated ideal MHD expectation. Nonetheless, the electron or generalized Walén test across this interval is acceptably compatible with the magnitude theoretically expected.

As an experimental aside, achieving fit agreement of the size of $\alpha_{\text {fit }}^{\prime}$ with that implied by the routine moment determinations, $\alpha_{\text {theory }}^{\prime}$, also indicates an upper limit of the possible absolute errors in the density and hence the mass density of the plasma. An upper limit for this absolute calibration is that the density cannot be in error of more than $\pm 2 \sigma_{\alpha_{e}} \approx 60 \%$ in the unlikely situation that all the disparity between $\alpha_{\text {fit }}^{\prime}$ and $\alpha_{\text {theory }}^{\prime}$ was attributable solely to the absolute calibration issues of the electrons; the incidence of other random errors such as aliasing of moment determination would reduce our in-flight estimate of this possible source of error.

\section{Misfits}

Figure 6 illustrates that examples may still be found, even with the generalized Walén test, where there is strong linear correlation suggested by the Walén relation but with the wrong slope. As the derivation above has emphasized, the two requirements for the existence of a relationship of this type are one-dimensional planar wave fronts in whose rest frame $\mathbf{E}_{T}$ is conserved (with $\mathbf{E}_{T} \simeq\left(\mathbf{E}_{M, e}\right)_{T}$ ) and the required Alfvén phase velocity of the wave along the normal. The poor electron fit could be rectified with a planar wave with a slower phase 

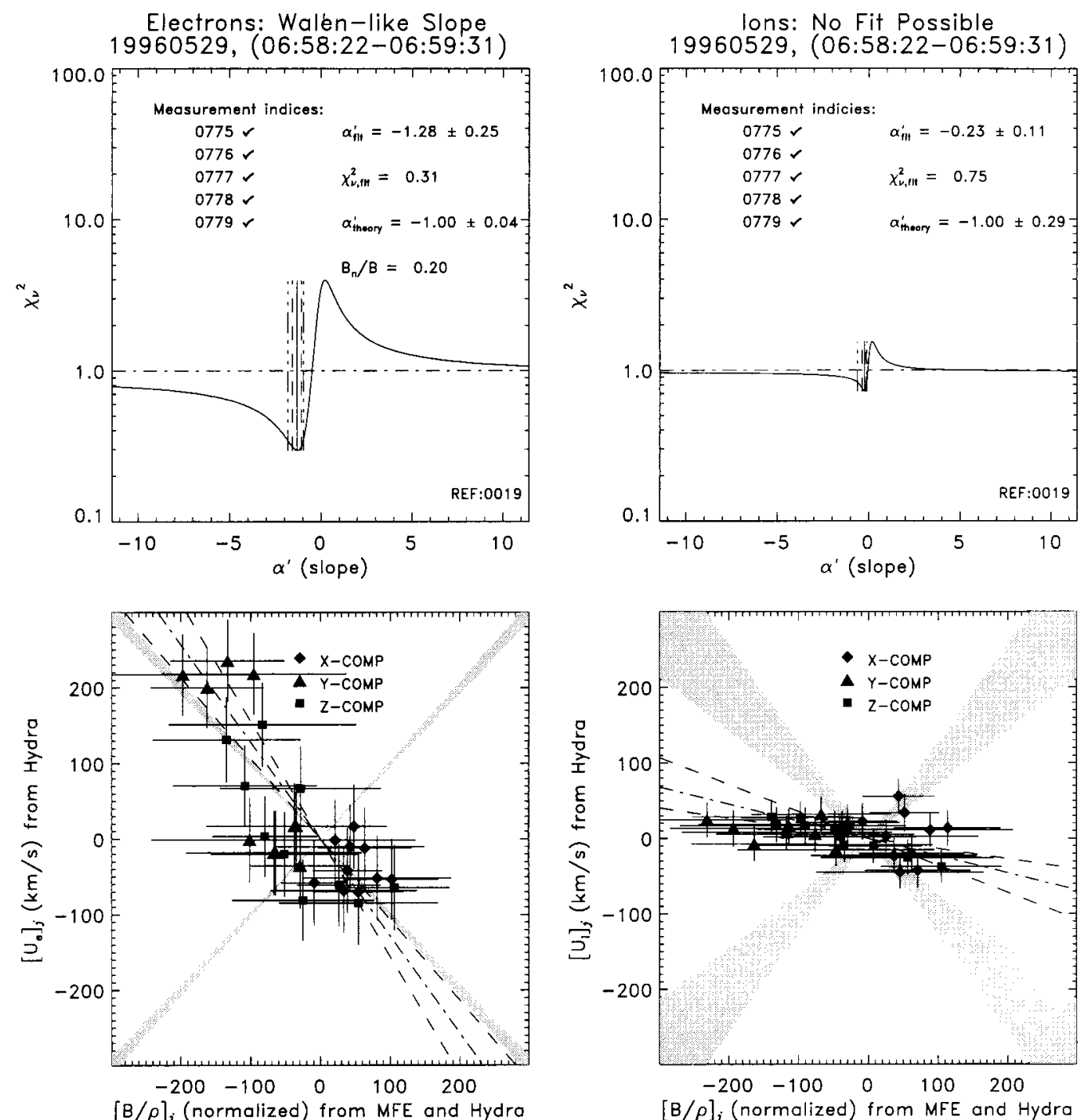

Figure 5. Walén fit $\chi^{2}$ surface, fit with error bounds for negative alpha.

velocity along $\mathbf{B}$. That such examples exist for electrons cannot be explained by just considering Alfvénic disturbances, unless corrections to $\left(\mathbf{E}_{M, e}\right)_{T}$ are important and or kinetic effects can modify the proper description of the Alfvén speed. Because the ions in this example do not meet the Walén expectation either and by about the same amount as the electrons, this example is yet a different possible explanation of a subset of poor Alfvénic comparisons of the type noted by Phan et al. [1996]. This type of analysis led us to reject this particular current shear region as a rotational discontinuity with $\mathbf{E}_{T}$ described only by $\left(\mathbf{E}_{M, e}\right)_{T}$ in (36)-(40).

\section{Internal Consistency and Cross-Checks Using $\mathbf{E}$ and $B$}

The actual normal flow direction of plasma in the laboratory frame need not be in the direction $U_{e, n}^{\prime} \hat{\mathbf{n}}$, since there is a potentially important motion of the free surface along the normal in the laboratory coordinate system, $V_{\mathrm{RD}, n}^{\mathrm{lab}}$, so that

$$
U_{e, n}^{\prime}=U_{e, n}^{\mathrm{lab}}-V_{\mathrm{RD}, n}^{\mathrm{lab}}
$$

Using conservation of mass in the rest frame of the RD, we can estimate the required, average laboratory speed of the RD surface along its normal:

$$
V_{\mathrm{RD}, n}^{\mathrm{lab}}=\left\langle\frac{\left[\rho_{\Sigma}(x) U_{e, n}^{\mathrm{lab}}(x)\right]}{\left[\rho_{\Sigma}(x)\right]}\right\rangle,
$$

where \langle\rangle$_{x}$ denotes an average over all distinct pairs.

For the observer moving with velocity $V_{\mathrm{RD}, n}^{\mathrm{ab}} \hat{\mathbf{n}}$, there should be a preserved constant tangential electric field $\mathbf{E}_{T}^{\prime}$ that may be estimated by the proxy relation

$$
c \mathbf{E}_{T}^{\prime} \simeq\left[-\left(\mathbf{U}_{e}^{\mathrm{lab}}-V_{\mathrm{RD}, n}^{\mathrm{lab}} \hat{\mathbf{n}}\right) \times \mathbf{B}(x)\right] \cdot(\widetilde{I}-\hat{\mathbf{n}} \hat{\mathbf{n}}),
$$

where $\simeq$ implies we have neglected the tangential contributions from $\left(\mathbf{E}-\mathbf{E}_{M, e}\right) \cdot(1-\hat{\mathbf{n}} \hat{\mathbf{n}})$ as being small. Finally, by transforming with a spatially constant transformation velocity $\mathbf{V}^{*}$ from the laboratory frame to a special [deHoffmann and Teller, 1950] frame, an observer perceives the electron flow and magnetic field to be parallel on either side and throughout the layer [Scudder et al., 1986] and subsequently reduces that observer's conserved tangential electric field to zero. This zero value should be approximated by the relation 

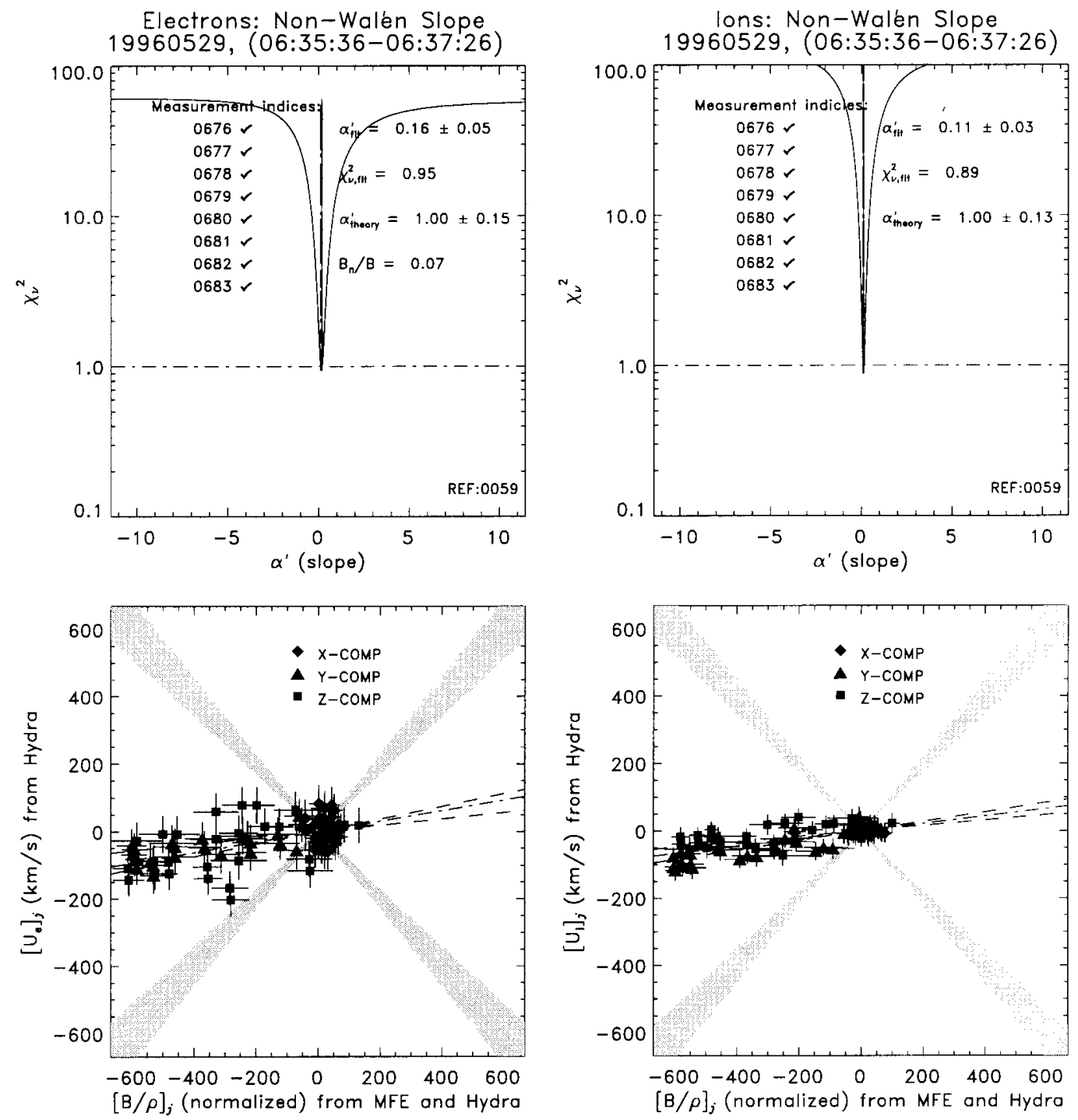

Figure 6. Unsuccessful Walén fit with error bounds.

$c \mathbf{E}_{T}^{\mathrm{HT}}=0 \simeq-\left[\left(\mathbf{U}_{e}^{\mathrm{lab}}-\mathbf{V}_{\mathrm{Hydra}}^{*}\right) \times \mathbf{B}(\mathbf{x})\right] \cdot(\widetilde{I}-\hat{\mathbf{n}} \hat{\mathbf{n}})$.

We have determined $\mathbf{V}_{\text {Hydra }}^{*}$ as a least squares problem that minimizes the sum of the square of the error normalized components of $\mathbf{E}_{T}^{\mathrm{HT}}$ from (48), namely,

$$
\chi_{\nu, \mathrm{HT}}^{2}\left(\mathbf{V}_{\mathrm{Hydra}}^{*}\right)=\sum_{j=1}^{N} \sum_{i=1}^{3}\left[\frac{E_{T, i}^{\mathrm{HT}}\left(t_{j}\right)}{\sigma_{i}\left(t_{j}\right)}\right]^{2},
$$

at the $N$ separate times, $t_{j}$, when three-dimensional electron moments were used in the Walén test. The denominators of (49) reflect the propagation of errors of the components and the flow that enter each component expression. With realistic errors, (49) should be $O$ (1) for an acceptable frame transformation velocity determination and if the proxy substitution of $\mathbf{E}_{T} \simeq\left(\mathbf{E}_{M, e}\right)_{T}$ is consistent with the data. (Note that for isotropic electrons the ambipolar contribution to $\mathbf{E}_{T}$ vanishes; thus the neglected terms are smaller than the normal components of $\mathbf{E}_{A, e}$ )

As a check within the fluid-magnetic field comparisons, we can assess the internal consistency of $\mathbf{V}_{\text {Hydra }}^{*}$ results by recog- nizing that its form can ultimately be cast in known, laboratory frame variables. We start by defining $\mathbf{V}_{\text {Hydra }}^{*}$ as

$$
\mathbf{V}_{\mathrm{Hydra}}^{*}=V_{\mathrm{RD}, n}^{\mathrm{lab}} \hat{\mathbf{n}}+\mathbf{U}_{e, T, j}^{\mathrm{lab}}+\mathbf{V}_{\mathrm{HT}, j}^{\mathrm{NIF}} .
$$

The subscript $j$ in the second and third terms on the right refers to the $j$ th measurement time. The first two terms of $\mathbf{V}_{\text {Hydra }}^{*}$ transform the laboratory observer into the (NIF) frame [Goodrich and Scudder, 1984], an RD rest frame where the plasma only flows in along the normal to the layer. The remaining part of (50) defines the further transformation velocity in that specific NIF RD rest frame to witness the layer in the deHoffmann-Teller frame. It is this latter quantity that is predicted in size and orientation to be

$$
\mathbf{V}_{\mathrm{HT}, j}^{\mathrm{NIF}}=\alpha \frac{\mathbf{B}_{T, j}^{\mathrm{lab}}}{\rho_{\Sigma, j}}
$$

which implies that

$$
\mathbf{V}_{\mathrm{Hydra}}^{*} \simeq V_{\mathrm{RD}, n}^{\mathrm{lab}} \hat{\mathbf{n}}+\mathbf{U}_{e, T, j}^{\mathrm{lab}}-\alpha \frac{\mathbf{B}_{T, j}^{\mathrm{lab}}}{\rho_{\Sigma, j}}
$$


As indicated, (50) is true for any time $t_{j}$ in the layer when ( $\mathbf{U}^{\text {lab }}$, $\mathbf{B}^{\text {lab }}$ ) are both known, since the null difference of any two versions of (50) at different times made in this way is automatically assured by the Walén relation itself. The agreement of $\mathbf{V}_{\text {Hydra }}^{*}$ (as determined from minimizing (49)) with the righthand side of (52), which we will call $\mathbf{V}_{\text {proxy }, j}^{*}$, is illustrated in Figure 7. Figure 7, left, uses only those successful Walén intervals, whereas Figure 7, right, uses only the unsuccessful Walén intervals. The first three plots in Figures 7, left and right, are for $j=1,2,3$ (see (50)) and the fourth plot superposes all components. These series of plots illustrate the statistical agreement between $\mathbf{V}_{\text {Hydra }}^{*}$ and $\mathbf{V}_{\text {proxy } j}^{*}$ for those intervals where the Walén condition was satisfied (Figure 7, left).

With the advent of three-axis electric field measurements, there is information to be had using $\mathbf{E}$ directly without using $\mathbf{E}_{M, e}$ of (36) as a proxy. The observed $\mathbf{E}$ has contributions, in principle, from all the terms in (36). In particular, $\mathbf{E}$ can have contributions along the normal and, in principle, transverse to it from the remaining terms in (36); the leading contribution expected would be $\mathbf{E}_{A, e}$ associated with the pressure gradient, the determinant of the deHoffmann-Teller electric field [Goodrich and Scudder, 1984].

As a further cross-check on the conclusions of electrons enforcing $\left[\mathbf{E}_{T}\right]=0$ within the RD layer, we have compared the values of the deHoffmann-Teller transformation velocity $V_{\text {EFI }}^{*}$ required to get from the spacecraft frame into the rotational discontinuity's deHoffmann-Teller rest frame using another method that minimizes the tangential electric field in the deHoffmann-Teller frame, namely, $\mathbf{E}_{T, \mathrm{EFI}}^{\mathrm{HT}}=\left(\mathbf{E}_{\mathrm{EFI}}+\mathbf{V}_{\mathrm{EFI}}^{*} \times\right.$ $\mathbf{B} / c) \cdot(\tilde{I}-\hat{\mathbf{n}} \hat{\mathbf{n}})$, which uses only the measured three-axis components of $\mathbf{E}_{\mathrm{EFI}}$ and $\mathbf{B}$ through the layer. (This approach is similar to that employed by Aggson et al. [1983] using two components of $\mathbf{E}$ from ISEE data. The different sensitivities of the respective axes of the EFI sensors are used in the minimization process. Figure 8 illustrates that differences in $\mathbf{V}^{*}$ determined by the two approaches are seen principally in the $x$ and $y$ GSM components. The error bars on the estimates are the result of least squares determinations for the best transformation speed as well as the $68 \%$ confidence level of recovering the three components of the transformation velocity in accordance with the standard practices [Press et al., 1992].

The present analysis of an approximate proxy for the electric field $c \mathbf{E}^{*}=-\mathbf{U}_{e} \times \mathbf{B}$ and the measured $\mathbf{E}_{\mathrm{EFI}}$ can, in principle, generate different deHoffmann-Teller frame transformation velocities. If corrections to $\mathbf{E}_{M, e}$ in the generalized Ohm's law for $\mathbf{E}$ in (36) are measurable in $\mathbf{E}_{\mathrm{EFI}}$, there could be an impact on $\mathbf{V}_{\mathrm{EFI}}^{*}$ that is present in Figure 8. Real differences between the two frames correspond to relaxation from EFI's "field line" preserving frame of reference to Hydra's "flux" preserving frame of reference [Scudder, 1997]. Clearly, in the absence of experimental error, $\mathbf{V}_{\mathrm{EFI}}^{*}$ is the more correct transformation to the deHoffmann-Teller frame.

To explore these differences, we examine in Figure 9 the residual deHoffmann-Teller electric field determined by the choice of $V_{\mathrm{EFI}}^{*}$ that minimizes the tangential component of the deHoffmann-Teller electric field. The results of this inspection may be summarized by the following: (1) the temporal average of the residual $\mathbf{E}_{\mathrm{EFI}}^{\mathrm{HT}}$ transverse to the minimum variance normal $\left(\mathbf{E}_{\mathrm{EFI}}^{T, \mathrm{HT}}\right)$ is below measurement error in almost all cases; (2) the residual HT electric field is measurable, along the minimum variance normal, and variable through the layer; (3) the magnitude of the time-averaged $\left|\langle\mathbf{E}\rangle_{t}^{\mathrm{HT}}\left(V_{\mathrm{EFI}}^{*}\right)\right|$ is power law correlated to the disagreement of $\mathbf{V}_{\mathrm{EFI}}^{*}$ and $\mathbf{V}_{\mathrm{Hydra}}^{*}$; and (4)
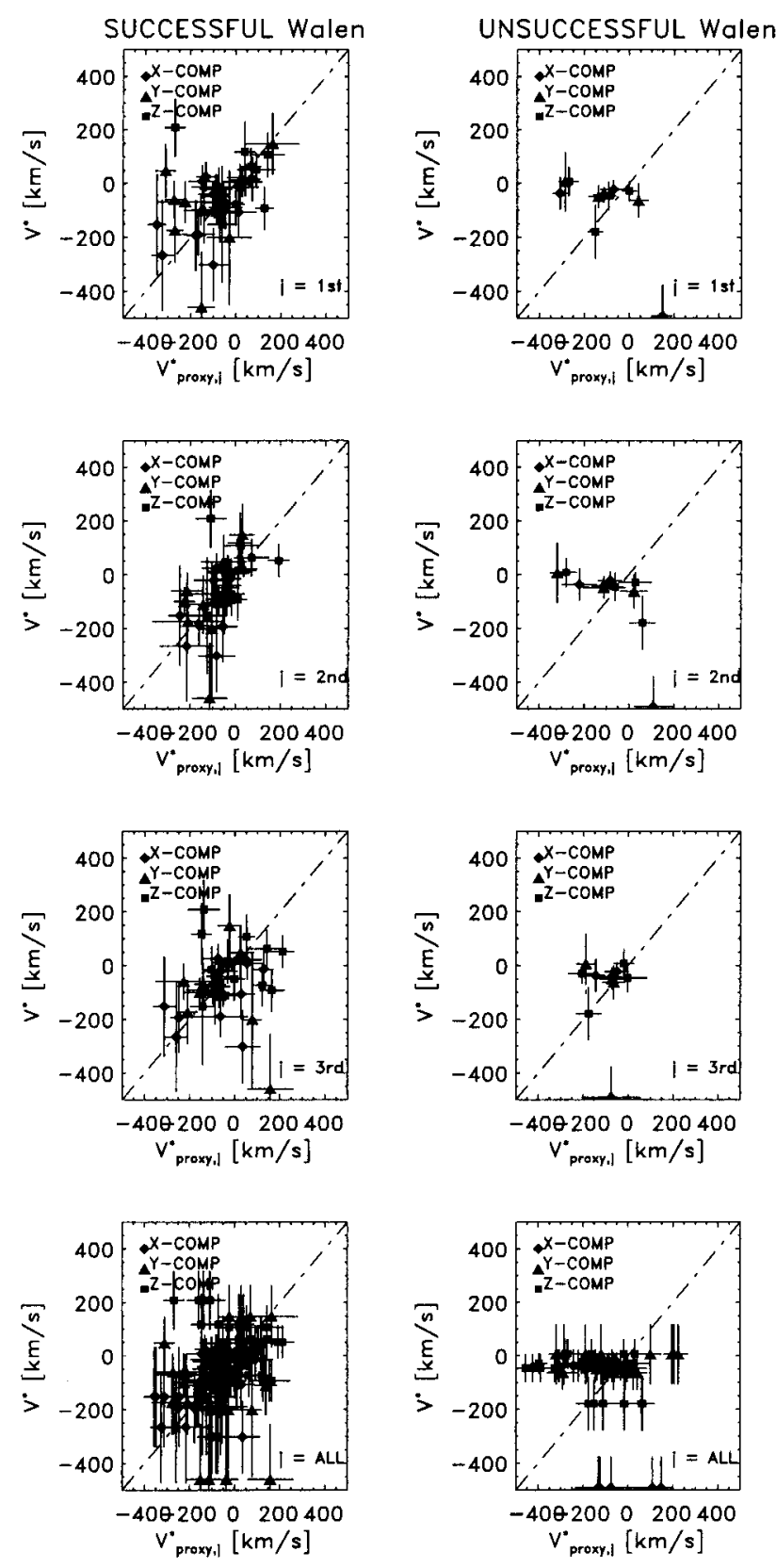

Figure 7. Velocity $\mathbf{V}_{\text {Hydra }}^{*}$ found from (49) plotted against $\mathbf{V}_{\text {proxy }}^{*}$ found from (23) for (left) successful and (right) unsuccessful Walén intervals.

the peak $\mathbf{E}(t)^{\mathrm{HT}}$ during each interval is indicated by the diamond in Figure 9 (top), while the trailing dashed line connects the peak to the average value discussed above. In this way we infer that some of the crossings encountered by Polar on this day contain effects of $\mathbf{E}_{A, e}$ and are direct reflections of the divergence of the electron pressure in these regions. These estimates represent the first empirical determinations of the order of magnitude $(0.3-5 \mathrm{mV} / \mathrm{m})$ of the deHoffmann-Teller electric fields along the normal in RDs in the magnetopause current layers. More comparisons of this type and the information that they contain in the geophysical matrix are discussed fully by J. D. Scudder et al. (manuscript in preparation, 1999). 

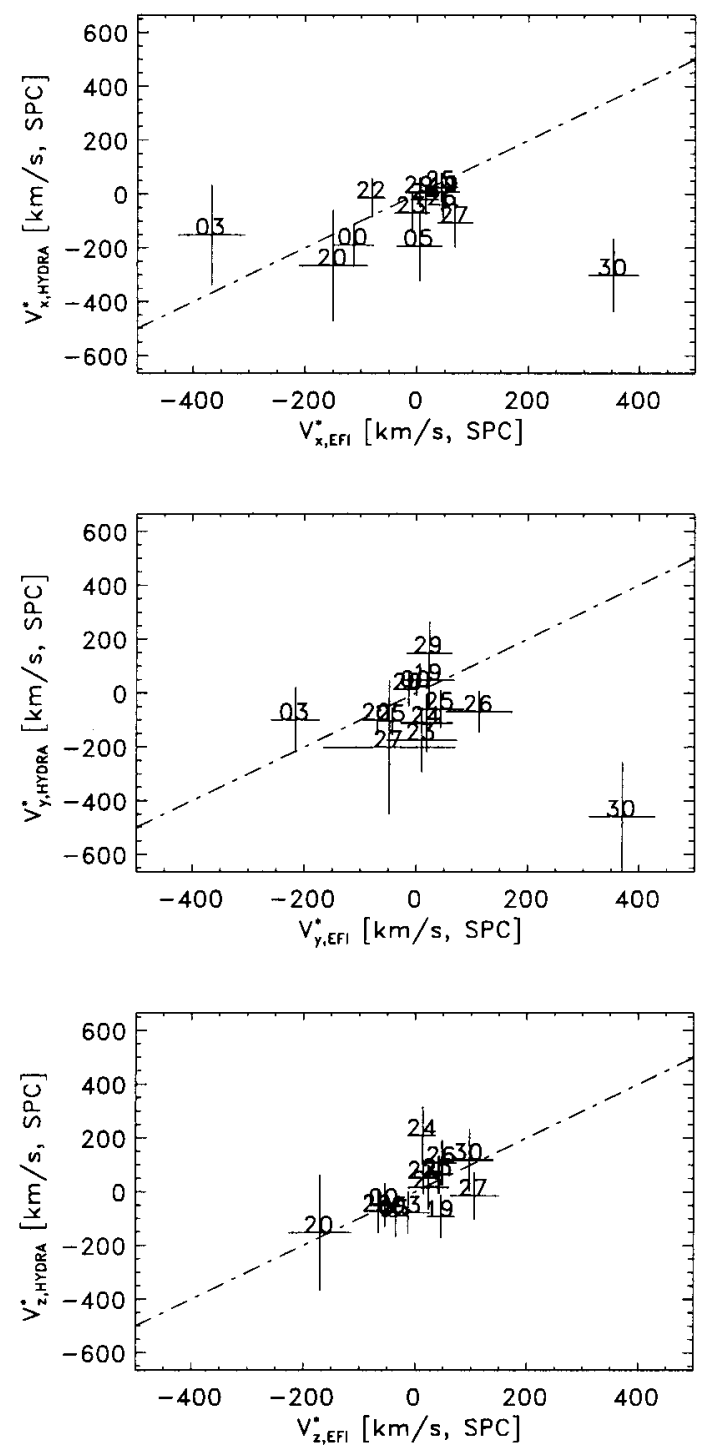

Figure 8. Comparison of deHoffmann-Teller transformation velocities determined using proxy $\mathbf{E}$ via $\mathbf{U}_{e}$ and $\mathbf{B}\left(\mathbf{V}_{\mathrm{Hydra}}^{*}\right)$ versus that from measured $\mathbf{E}$ and $\mathbf{B}\left(\mathbf{V}_{\mathrm{EFI}}^{*}\right)$.

\section{Statistical Comparison of Ion and Electron Estimates of $\boldsymbol{R}_{\boldsymbol{A}}$}

To establish contact with the previous statistical work with ions of Paschmann et al. [1979] and Phan et al. [1996], we next contrast new statistical work with Polar electron and ion flow measurements and field data at current layers at the Earth's magnetopause. We will summarize our attempts to verify the Walén test in the electron form of (41) and contrast them with the Hydra ion measurements to test (1) through the same layer. We reemphasize that the basic distribution function snapshot for electrons and ions is acquired in an interleaved way, so that the net time aliasing between the ion moments and their electron counterparts is $1.15 \mathrm{~s}$ of the total $13.8 \mathrm{~s}$. Since (41) is only correct for the electrons and differs from the same statement for ions by terms that involve the current density (compare with (43b)), systematic differences between the value of the proportionality constant $\alpha_{e}$ from the electron fit to (43b) and that of the proxy slope $\alpha_{i}$ by fitting the ions to (1) could be a reflection of the fact that ions cannot follow the magnetic field as agilely as the electrons, who in the deHoffmann-Teller frame precisely "carry" the magnetic tube of force from one side of the layer to its asymptotic location on the other side, while the ions "miss" part of the turns and end up jumping field lines, resulting in cross-field currents [Scudder, 1987, manuscript in preparation, 1999].

Accordingly, the ion inference of the effective Walén proportionality constant $|\alpha|$ should always be smaller than that using the electrons as illustrated in specific case studies in Figures 3-5. Figure 10 (top) contrasts fit slopes for all 44 events in which both electron and ion regressions to straight lines had boundable errors with 68\% confidence. Figure 10 (bottom) illustrates only those electron fits with "Walén-like" slopes (in the sense of (41) with $\left.\alpha_{e}^{\prime}= \pm(1 \pm \sigma)\right)$ to illustrate the corresponding values of $\alpha_{i}^{\prime}$ when analyzing the same interval in time. The only proviso for inclusion in Figure 10 (top) was that an ion fit was an acceptable approximation to a linear relation. Most of the ion slopes in Figure 10 (top) are well removed from unity. The set of points indicated in the solid squares represent those two events (5\%) of the class of 38 in Figure 10 where ions and electrons simultaneously have good Walén-like fits. Figure 10 (bottom) considers the broader class of all 44 events where linear fits to electrons and ions in the same interval were acceptable characterizations of the data. The first observation is the widespread tendency for the electron slopes to hover at and around $\left|\alpha_{e}^{\prime}\right| \simeq 1$. Detailed examples of $\alpha_{e}^{\prime} \simeq$ $\pm 1, \simeq 0$ are given in Figures 3-5. Of the augmented class of all simultaneous linear fits with boundable errors, only 2 of 44
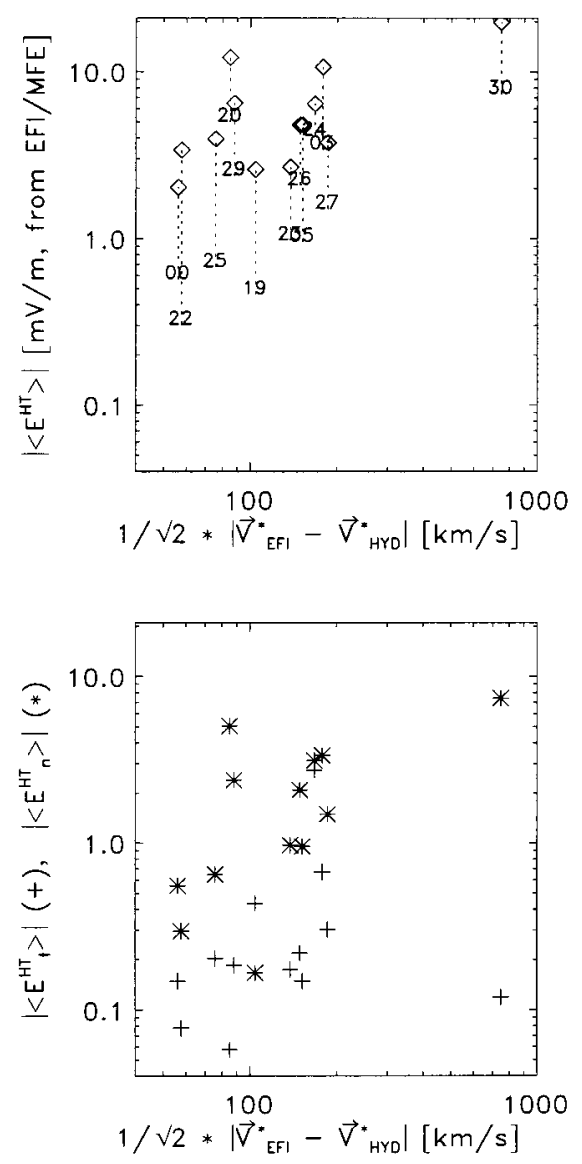

Figure 9. Examples of how $\mathbf{E}^{H T}$ scales with $\sqrt{ } \overline{\Sigma_{j} \delta_{j}^{2}}=$ $\left|\mathbf{V}_{\mathrm{EFI}}^{*}-\mathbf{V}_{\mathrm{Hydra}}^{*}\right| / \sqrt{2}$. 
( $\simeq 4.5 \%)$ were acceptable from the ion perspective. Restated, $95 \%$ of the ion proxy fits had proportionality constants that were systematically lower than the jump conditions would require (compare Figures 4 and 5). The average values for $\alpha_{i}^{\prime}$ in Figure 10, top and bottom, are 0.39 and 0.37 , respectively. The restricted average of those cases where $\alpha_{i}^{\prime}>0.5$ was 0.63 . The precise value of the mean is undercut by the wide variance seen in Figure 10. This ion behavior and smaller than unity size for $\alpha_{i}^{\prime}$ are precisely the morphology reported in the statistical work using the Active Magnetospheric Particle Tracer Explorers Ion Release Module (AMPTE IRM) data [Phan et al., 1996]. These circumstances are also reminiscent of those reported for interplanetary Alfvén wave trains where Belcher et al. [1969] determined an ion Alfvén ratio that is too small.

We understand this systematic effect by noting that (41) is not properly used for ions within the current-carrying layer of these structures (compare (43b)), even using the center of mass velocity of the ions, since the Walén condition in terms of the center of mass is only expected for asymptotic jump conditions (15) when the current-carrying layer is no longer present during the moment measurements on either side of it.

There is, additionally, a subtlety to the issue that this effect might be present in wave trains that, with periods of hours, are arguably very long wavelength. For a single, large-amplitude, long-wavelength mode the current corrections in (43b) might be argued to be imperceptibly small. In the presence of a large-amplitude Alfvénic disturbance a spectrum of wavenumbers is invariably present as illustrated by Belcher et al. [1969]. A companion paper (J. D. Scudder, manuscript in preparation, 1999) demonstrates with simulations and observations that the effects of the omnipresent currents are present no matter what the scale length of the dominant wavelength, provided "interesting" inertial scale structures are superposed. These are observed within the interplanetary Alfvénic wave trains [Belcher and Solodyna, 1975], and the magnetopause current layers are rich with such ion inertial scale structures (J. D. Scudder et al., manuscript in preparation, 1999).

We have analytically demonstrated in this paper that essentially the same constant from the asymptotic Walén jump conditions is the correct constant for use with the generalized Walén test with electron bulk velocity changes within the layer (see (33c) and (33d)). We have also shown experimentally that the electrons meet the expected generalized Walén proportionality constant values derived in (33a)-(33f) more often $(>95 \%)$ than do the ions when the tests are performed over data that are collected in an interleaved manner and aliased over the same total time.

The only test of the Walén form that is "well posed" when using points within extended, finite amplitude wave trains or more localized current layers (wave packets with many k) as in the present paper is the electron version (41). Conversely, it would appear from comparing (43b) and (33a) that it is difficult to infer what the implications are of the proxy ion data when the data used do not meet the expected $(\mathbf{J}=0)$ asymptotic conditions. In this sense the Walén proxy "test" assuming the form (1) using ions including particles within the rotation of $\mathbf{B}$ current-carrying layer is a "broken lever" and is, at best, a qualitative measure of the existence of a rotational discontinuity. That the ion fit values do not agree with that expected from (1) cannot be inverted to imply new physics about momentum transfer.
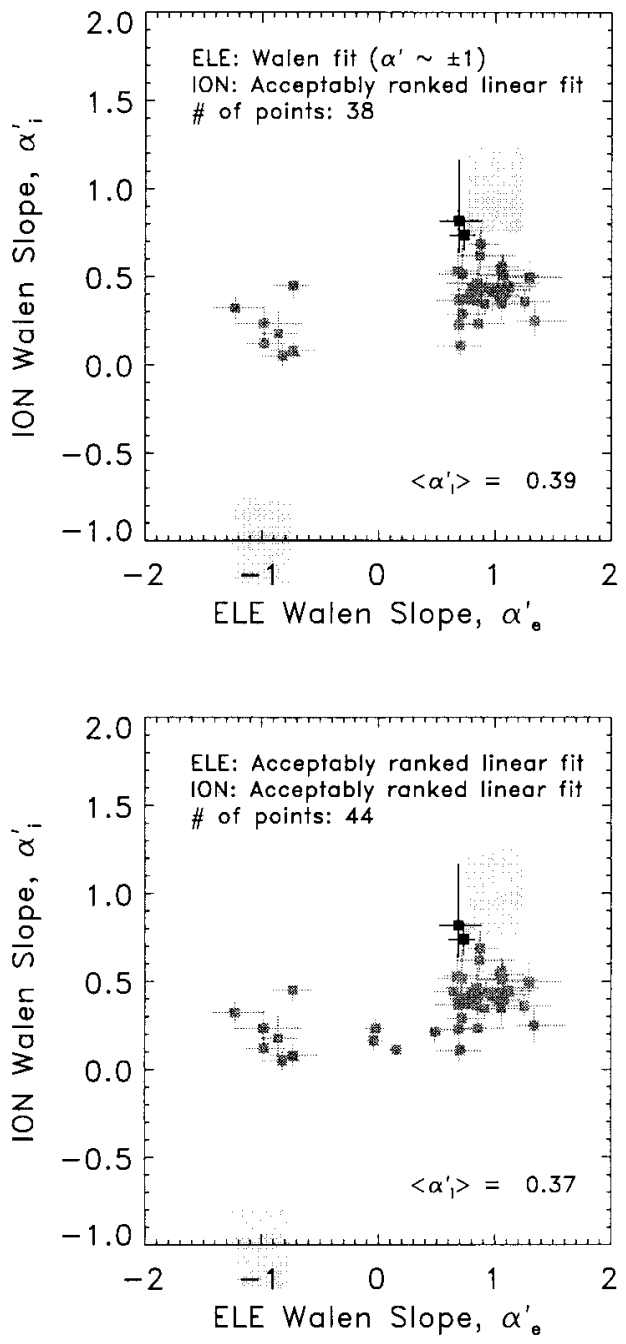

Figure 10. Contrast of ion proxy scaled slopes $\alpha_{i}^{\prime}$ versus the electron scaled slopes $\alpha_{e}^{\prime}$.

\section{Discussion}

We have illustrated that the electron fluid velocity is a succinct digest of the twisting of the magnetic field within an Alfvénic wave train or "inside" a rotational discontinuity and have generalized the Walén test in the form of (41) to reflect this understanding. We have illustrated the utility of this test with three-dimensional electron moment parameters, the magnetometer and electric field observations at 44 incursions of current carrying layers within Earth's magnetopause. The observed electron form of these tests are in excellent agreement with the theory, even though current layers are being penetrated, if not traversed, and with no attempt to edit data points as being outside or inside the current layer. Successfully fitting the data to the generalized Walén relation does not require nor certify traversal of the magnetopause proper, only penetration of the current-carrying layer of an Alfvénic wave train or rotational discontinuity.

On the Polar orbit of May 29, 1996, where the data of this paper were collected, there were 44 acceptable electron Walén Alfvén current layer penetrations of the quality of Figures 3 and 4. Many of the previous "good" ion Walén tests at the subsolar magnetopause [Phan et al., 1996] reported over the years have the slope of the regression $\alpha_{i}$ between (ion) flow 
and field jumps as small as $50 \%$ of that expected. Of our successful electron tests, $95 \%$ of the ion proxy tests (using the same time intervals as the electrons) through the layer had Walén slopes smaller than their electron counterparts. From the vantage point of the generalized Ohm's law this can be understood as the ions not matching the electron's dexterity within the current layer in following the magnetic field rotations. This property is well known in the two-fluid description of an Alfvén wave [Chen, 1990] and is discussed more completely in the case of circular polarization in the companion paper (J. D. Scudder, manuscript in preparation, 1999).

It is also possible that the layers that fail the electron "Walén" test based on (41) are current-carrying boundary layers across which there is shear, as in a tangential discontinuity, whose shear is a remnant of some preexisting dynamical alignment. This situation occurs in the solar wind across many tangential discontinuities (TDs) [Burlaga, 1971]. The dynamical coherence of the changes in $\mathbf{U}$ and $\mathbf{B}$ across these regions led early researchers [Belcher and Davis, 1971] to classify these as rotational discontinuities, even though they were often subsequently shown to be TDs. Interestingly, the Belcher and Davis arguments were that the chance of fortuitous vector correlation was poor, so the lack of precise proportionality constant was viewed as an annoyance. At the magnetopause, until the present observations, we had a similar argument, where the vector correlations were thought to be good but the proportionality factors were not as expected. At the magnetopause there are always those who would claim the TD is the correct alternative! Thus the present measurements are an important quantitative anchor in the case for collisionless reconnection.

Another possibility, though remote, is that the mass density or some other factor in the theoretically required constants in $\alpha$ are not empirically well measured. In the example of Figure 6 this would require the mass density to be 100 times that measured to correct, by itself, the disagreement between measurements and observations. For the present Polar Hydra observations the ion and electron densities agree (without intracalibration [Scudder et al., 1999]) through these structures at better than $10 \%$; the minor missing ion explanation does not seem tenable. This is particularly clear when it would appear that the easiest way to change the effective mass in the proportionality constant is to change the ion compositional mix. Given that the electron charge density as observed already covers the ion charge density on the assumption that they are protons, this does not seem to be a viable avenue to approach the discrepancy.

Examples of structures that do not satisfy the generalized Alfvénic form of the Walén condition (based on $\mathbf{E} \simeq \mathbf{E}_{M, e}$ ) were also illustrated that could be understood as planar waves with slower phase velocities than the Alfvén branch, such as the slow mode.

There is the tendency for interplanetary RDs to be found embedded in a background of much lower frequency Alfvénic background punctuated with ion inertial scale length structures that contradict the theoretical requirement for the asymptotic readings of the RD to be outside of all the causes for the twisting of the field and the fluid. Precursor and trailing wave trains are, nevertheless, present in the observations used to define the asymptotic, "current free" states, much like are seen in modern simulations [Krauss-Varban et al., 1995]. With these systematics the issue of the existence of RDs as isolated structures between theoretically idealized, gradient free asymptotic states may represent such a severe idealization that asymptotic jumps may not be experimentally available. If this is the situation, the failure of the ion tests to obtain the correct proportionality constant as has been previously reported may be related to the same issues that motivate our examination of the postideal MHD considerations that identify the electrons as the best tests of the "Alfvénicity."

Finally, there is a distinct possibility that the appropriate Walén condition for very thin layers could require inclusion of the ambipolar electric field's contribution, $\mathbf{E}_{A, e}$ to the tangential electric field, even for a one-dimensional layer in the presence of pressure anisotropy within the layer. This is a viable possibility, only to the extent that there is nonzero electron pressure anisotropy, since, otherwise, this term does not contribute to $\mathbf{E}_{T}$.

The advent of the electron Walén level layer diagnostic (41) for Alfvénic wave trains and RDs carries with it the benefits of the $\mathbf{k}$ vector geometry. The price for using this diagnostic is the careful exclusion of photoelectrons, correction for spacecraft potential modifications, and careful numerical integrations over subsonic electron distribution functions. As guided by theory, taking such care results in a very nice diagnostic of the rearrangement of field lines, which has been hitherto completely unavailable and will soon be used to delineate the topology within reconnection layers available to the Polar spacecraft (J. D. Scudder et al., manuscript in preparation, 1999). Along the way, these results appear to resolve the puzzle of the lack of full Walén proportionality in the previous ion proxy tests for a substantial fraction of actual RDs at the magnetopause and a longstanding similar issue for interplanetary rotational discontinuities (J. D. Scudder, manuscript in preparation, 1999).

Acknowledgments. It is a pleasure to acknowledge the helpful comments of the referees. The present results of the Hydra investigation were made possible by the decade-long hardware efforts of groups led at NASA GSFC by Keith Ogilvie, at UNH by Roy Torbert, at MPAE by Axel Korth, and at UCSD by W. Fillius. We would also like to acknowledge the innovative software development by R. D. Holdaway and J. B. Faden at the University of Iowa. The funding research for this paper at University of Iowa, UC Berkeley, and UCLA was performed under grants $(\mathrm{G})$ or contracts $(\mathrm{C})$ to NASA, NAG52231(G), NAG5-3171(C), and NAG5-3182(C). The German support for Hydra activities is via DARA grant $500 \mathrm{C} 89110$.

Janet G. Luhmann thanks Johan DeKeyser and Stephen A. Fuselier for their assistance in evaluating this paper.

\section{References}

Aggson, T. L., P. J. Gambardella, and N. C. Maynard, Electric field measurements at rotational magnetopause discontinuities, J. Geophys. Res., 88, 10,000, 1983.

Belcher, J. W., and L. Davis, Large amplitude Alfvén waves in the interplanetary medium, 2, J. Geophys. Res., 76, 3534, 1971.

Belcher, J. W., and C. V. Solodyna, Alfvén waves and directional discontinuities in the interplanetary medium, J. Geophys. Res., 80, 181, 1975.

Belcher, J. W., J. L. Davis, and E. Smith, Large amplitude Alfvén waves in the interplanetary medium: Mariner 5, J. Geophys. Res., 74, 2302, 1969.

Burlaga, L. F., Nature and origin of directional discontinuities in the solar wind, J. Geophys. Res., 76, 4360, 1971.

Chen, F. F., Plasma Physics and Controlled Fusion, 2nd ed., Plenum, New York, 1990.

Cowley, S. W. H., Plasma populations in a simple open magnetosphere, Space Sci. Rev., 26, 217, 1980.

deHoffmann, F., and E. Teller, Magnetohydrodynamic shocks, Phys. Rev., 80, 692, 1950. 
DeKeyser, J., M. Roth, and A. Soding, Flow shear across solar wind discontinuities: Wind observations, Geophys. Res. Lett., 25, 2649, 1998.

Goodrich, C. C., and J. D. Scudder, The adiabatic energy change of plasma electrons and the frame dependence of the cross shock potential at collisionless magnetosonic shock waves, J. Geophys. Res., 89, 6654, 1984.

Harvey, P., et al., The GGS/Polar magnetic fields investigation, in The Global Geospace Mission, edited by C. Russell, p. 583, Kluwer Acad., Norwell, Mass., 1995.

Hudson, P. D., Discontinuities in anisotropic plasma and their identification in the solar wind, Planet. Space Sci., 18, 1611, 1970.

Hudson, P. D., Rotational discontinuities in an anisotropic plasma, Planet Space Sci., 19, 1693, 1971.

Krauss-Varban, D., H. Karimabadi, and N. Omidi, Kinetic structure of rotational discontinuities: Implications for the magnetopause, $J$. Geophys. Res., 100, 11,981, 1995.

Levy, R. H., H. E. Petschek, and G. L. Siscoe, Aerodynamic aspects of the magnetospheric flow, AIAA J., 2, 2065, 1964.

Neugebauer, M., D. R. Clay, B. E. Goldstein, B. Tsuritani, and R. Zwickl, A reexamination of rotational and tangential discontinuities in the solar wind, J. Geophys. Res., 89, 5395, 1984.

Parker, E. N., Newtonian development of the dynamic properties of ionized gases of low density, Phys. Rev., 107, 924, 1957.

Paschmann, G., et al., Plasma acceleration at the earth's magnetopause: Evidence for reconnection, Nature, 282, 243, 1979.

Phan, T.-D., G. Paschmann, and B. U. O. Sonnerup, Low-latitude dayside magnetopause and boundary layer for high magnetic shear, 2, Occurrence of magnetic reconnection, J. Geophys. Res., 101, 7817, 1996.

Press, W. H., S. Teukolsky, W. T. Bettering, and B. Flannery, Numerical Recipes, 2nd ed., Cambridge Univ. Press, New York, 1992.

Rossi, B., and S. Olbert, Introduction to the Physics of Space, McGrawHill, New York, 1970.

Russell, C. T., R. C. Snare, J. D. Means, D. Pierce, D. Dearborn, M. Larson, G. Barr, and G. Le, The GGS/Polar magnetic fields investigation, in The Global Geospace Mission, edited by C. Russell, p. 563, Kluwer Acad., Norwell, Mass., 1995.

Scudder, J. D., The field-aligned flow approximation for electrons within layers possessing a normal mass flux: A corollary to the deHoffmann Teller theorem, J. Geophys. Res., 92, 13,447, 1987.

Scudder, J. D., Theoretical approaches to the description of magnetic merging: The need for finite $\beta_{e}$, anisotropic ambipolar Hall MHD, Space Sci. Rev., 80, 235, 1997.

Scudder, J. D., A. Mangeney, C. Lacombe, C. Harvey, T. L. Aggson, R. Anderson, J. T. Gosling, G. Paschmann, and C. T. Russell, The resolved layer of a collisionless, high $\beta$, supercritical, quasiperpendicular shock wave, 1, Rankine Hugoniot geometry, currents, and stationarity, J. Geophys. Res., 91, 11,019, 1986.

Scudder, J. D., et al., Hydra-A 3-dimensional electron and ion hot plasma instrument for the Polar spacecraft of the GGS mission, in The Global Geospace Mission, edited by C. Russell, p. 459, Kluwer Acad., Norwell, Mass., 1995.

Scudder, J. D., X. Cao, and F. S. Mozer, The photoemission currentspacecraft voltage relation: Key to routine, quantitative low-energy plasma measurements, J. Geophys. Res., in press, 1999.

Sonnerup, B. U. O., G. Paschmann, I. Papamastorakis, N. Sckopke, G. Haerendel, S. J. Bame, J. R. Asbridge, J. T. Gosling, and C. T. Russell, Evidence for magnetic field reconnection at the earth's magnetopause, J. Geophys. Res., 86, 10,049, 1981.

Sonnerup, B. U. O., I. Papamastorakis, G. Paschmann, and H. Luhr, Magnetopause properties from AMPTE-IRM observations of the convection electric field: Method development, J. Geophys. Res., 92, 12,137, 1987.

Sonnerup, B. U. O., I. Papamastorakis, G. Paschmann, and H. Luhr, The magnetopause for large magnetic shear: Analysis of convection electric fields from AMPTE-IRM, J. Geophys. Res., 95, 10,541, 1990. Walén, C., On the theory of sunspots, Ark. Mat. Astron. Fys., 30A, 15, 1944.

F. S. Mozer, Space Sciences Laboratory, University of California, Berkeley, CA 94720.

K. W. Ogilvie, Laboratory for Extraterrestrial Physics, NASA Goddard Space Flight Center, Code 690, Greenbelt, MD 20770.

P. Puhl-Quinn and J. D. Scudder, Department of Physics and Astronomy, University of Iowa, Van Allen Hall, Room 203, Dubuque at Jefferson Streets, Iowa City, IA 52242.(jds@space-theory.physics. uiowa.edu)

C. T. Russell, Institute of Geophysics and Planetary Physics, University of California, Los Angeles, CA 90024.

(Received September 25, 1998; revised February 22, 1999; accepted March 15, 1999.) 
